\title{
Left-symmetric bialgebroids and their corresponding Manin triples *
}

\author{
Jiefeng Liu ${ }^{1}$, Yunhe Sheng ${ }^{1,2}$ and Chengming Bai ${ }^{3}$ \\ ${ }^{1}$ Department of Mathematics, Xinyang Normal University, \\ Xinyang 464000, Henan, China \\ ${ }^{2}$ Department of Mathematics, Jilin University, \\ Changchun 130012, Jilin, China \\ ${ }^{3}$ Chern Institute of Mathematics and LPMC, Nankai University, \\ Tianjin 300071, China \\ Email: jfliu12@126.com; shengyh@jlu.edu.cn; baicm@nankai.edu.cn
}

\begin{abstract}
In this paper, we introduce the notion of a left-symmetric bialgebroid as a geometric generalization of a left-symmetric bialgebra and construct a left-symmetric bialgebroid from a pseudo-Hessian manifold. We also introduce the notion of a Manin triple for left-symmetric algebroids, which is equivalent to a left-symmetric bialgebroid. The corresponding double structure is a pre-symplectic algebroid rather than a left-symmetric algebroid. In particular, we establish a relation between Maurer-Cartan type equations and Dirac structures of the pre-symplectic algebroid which is the corresponding double structure for a left-symmetric bialgebroid.
\end{abstract}

\section{Introduction}

Left-symmetric algebras (or pre-Lie algebras) arose from the study of convex homogeneous cones [27, affine manifolds and affine structures on Lie groups 10, deformation and cohomology theory of associative algebras [6] and then appear in many fields in mathematics and mathematical physics. See the survey article [2] and the references therein. In particular, there are close relations between left-symmetric algebras and certain important left-invariant structures on Lie groups like aforementioned affine, symplectic, Kähler, and metric structures [8, 11, 17, 19, 20. A quadratic left-symmetric algebra is a left-symmetric algebra together with a nondegenerate invariant skewsymmetric bilinear form [3]. A symplectic (Frobenius) Lie algebra is a Lie algebra $\mathfrak{g}$ equipped with a nondegenerate 2-cocycle $\omega \in \wedge^{2} \mathfrak{g}^{*}$. There is a one-to-one correspondence between symplectic (Frobenius) Lie algebras and quadratic left-symmetric algebras.

\footnotetext{
${ }^{0}$ Keywords: left-symmetric algebroid, left-symmetric bialgebroid, pseudo-Hessian manifold, pre-symplectic algebroid, Manin triple

${ }^{0}$ MSC: 17B62,53D12,53D17,53D18

${ }^{*}$ This research is supported by NSF of China $(11471139,11271202,11221091,11425104)$, SRFDP (20120031110022) and NSF of Jilin Province (20140520054JH).
} 
A left-symmetric algebroid, also called a Koszul-Vinberg algebroid, is a geometric generalization of a left-symmetric algebra. See [12, 22, 23] for more details and applications. In [13], we introduced the notion of a pre-symplectic algebroid, which is a geometric generalization of a quadratic leftsymmetric algebra. Generalizing the relation between symplectic (Frobenius) Lie algebras and quadratic left-symmetric algebras, we showed that there is a one-to-one correspondence between symplectic Lie algebroids and pre-symplectic algebroids. See [4, 7, 18, 21] for more details about symplectic Lie algebroids and their applications.

The purpose of this paper is studying the bialgebra theory for left-symmetric algebroids and the corresponding Manin triple theory. Motivated by [14, 16, we introduce the notion of a left-symmetric bialgebroid, which is a geometric generalization of a left-symmetric bialgebra [1]. The double of a left-symmetric bialgebroid is not a left-symmetric algebroid anymore, but a presymplectic algebroid. This result is parallel to the fact that the double of a Lie bialgebroid 1 , is not a Lie algebroid, but a Courant algebroid [14]. Furthermore, if we consider the commutator of a left-symmetric bialgebroid, we can obtain a matched pair of Lie algebroids, whose double is the symplectic Lie algebroid associated to the pre-symplectic algebroid. The above results can be summarized into the following commutative diagram:

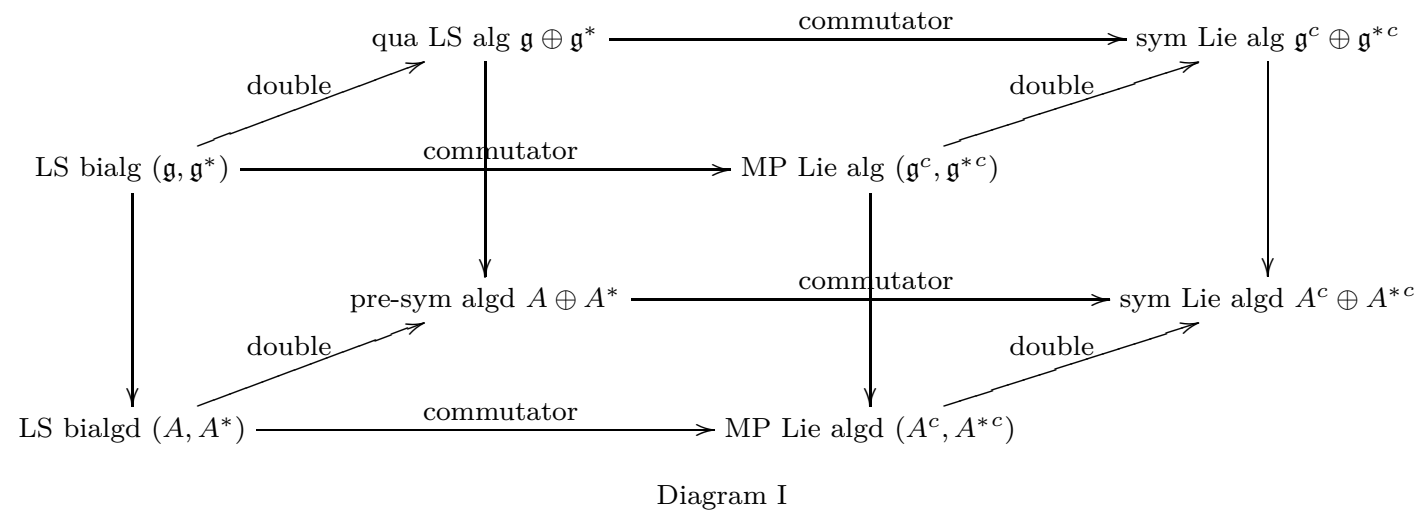

In the above diagram, qua is short for quadratic, LS is short for left-symmetric, alg is short for algebra, sym is short for symplectic, MP is short for matched pair and algd is short for algebroid. We establish a relation between left-symmetric bialgebroids and pseudo-Hessian manifolds. See 24, 25, 26] for more information about pseudo-Hessian Lie algebras and Hessian geometry. A flat manifold $(M, \nabla)$ gives rise to a left-symmetric algebroid $T_{\nabla} M$. We show that a pseudo-Riemannian metric $g$ on $(M, \nabla)$ is a pseudo-Hessian metric if $\delta g=0$, where $\delta$ is the cohomology operator of the left-symmetric algebroid $T_{\nabla} M$ (Proposition 4.12). Given a pseudo-Hessian manifold $(M, \nabla, g)$, $\left(T_{\nabla} M, T_{H}^{*} M\right)$ is a left-symmetric bialgebroid (Proposition 4.13), where $H$ is the inverse of $g$. This result is parallel to that $\left(T M, T_{\pi}^{*} M\right)$ is a Lie bialgebroid for any Poisson manifold $(M, \pi)[9,16$. It seems that our theory is a symmetric analogue of Poisson geometry.

The paper is organized as follows. In Section 2, we give a review on Lie algebroids, leftsymmetric algebroids and pre-symplectic algebroids. In Section 3, we develop the differential calculus on a left-symmetric algebroid which is the main tool in our later study. In Section 4, we introduce the notion of a left-symmetric bialgebroid and study its properties. In Section 5, we introduce the notion of a Manin triple for left-symmetric algebroids and show the equivalence between left-symmetric bialgebroids and Manin triples for left-symmetric algebroids.

\footnotetext{
${ }^{1}$ The notion of a Lie bialgebroid was first introduced by Mackenzie and $\mathrm{Xu}$ in as the infinitesimal object of a Poisson groupoid [16].
} 
Throughout this paper, all the vector bundles are over the same manifold $M$. For two vector bundles $A$ and $B$, a bundle map from $A$ to $B$ is a base-preserving map and $C^{\infty}(M)$-linear.

Acknowledgement: We give our warmest thanks to Zhangju Liu and Jianghua Lu for very useful comments and discussions.

\section{Preliminaries}

We briefly recall Lie algebroids, left-symmetric algebroids and pre-symplectic algebroids.

\section{Lie algebroids}

The notion of a Lie algebroid was introduced by Pradines in 1967, which is a generalization of Lie algebras and tangent bundles. See [15] for general theory about Lie algebroids. They play important roles in various parts of mathematics.

Definition 2.1. A Lie algebroid structure on a vector bundle $\mathcal{A} \longrightarrow M$ is a pair that consists of a Lie algebra structure $[\cdot, \cdot]_{\mathcal{A}}$ on the section space $\Gamma(\mathcal{A})$ and a bundle map $a_{\mathcal{A}}: \mathcal{A} \longrightarrow$ TM, called the anchor, such that the following relation is satisfied:

$$
[x, f y]_{\mathcal{A}}=f[x, y]_{\mathcal{A}}+a_{\mathcal{A}}(x)(f) y, \quad \forall f \in C^{\infty}(M) .
$$

For a vector bundle $E \longrightarrow M$, we denote by $\mathfrak{D}(E)$ the gauge Lie algebroid of the frame bundle $\mathcal{F}(E)$, which is also called the covariant differential operator bundle of $E$.

Let $\left(\mathcal{A},[\cdot, \cdot]_{\mathcal{A}}, a_{\mathcal{A}}\right)$ and $\left(\mathcal{B},[\cdot, \cdot]_{\mathcal{B}}, a_{\mathcal{B}}\right)$ be two Lie algebroids (with the same base), a basepreserving morphism from $\mathcal{A}$ to $\mathcal{B}$ is a bundle map $\sigma: \mathcal{A} \longrightarrow \mathcal{B}$ such that

$$
a_{\mathcal{B}} \circ \sigma=a_{\mathcal{A}}, \quad \sigma[x, y]_{\mathcal{A}}=[\sigma(x), \sigma(y)]_{\mathcal{B}} .
$$

A representation of a Lie algebroid $\mathcal{A}$ on a vector bundle $E$ is a base-preserving morphism $\rho$ form $\mathcal{A}$ to the Lie algebroid $\mathfrak{D}(E)$. Denote a representation by $(E ; \rho)$. The dual representation of a Lie algebroid $\mathcal{A}$ on $E^{*}$ is the bundle map $\rho^{*}: \mathcal{A} \longrightarrow \mathfrak{D}\left(E^{*}\right)$ given by

$$
\left\langle\rho^{*}(x)(\xi), u\right\rangle=a_{\mathcal{A}}(x)\langle\xi, u\rangle-\langle\xi, \rho(x)(u)\rangle, \quad \forall x \in \Gamma(\mathcal{A}), \xi \in \Gamma\left(E^{*}\right), u \in \Gamma(E) .
$$

As a generalization of a matched pair of Lie algebras, a matched pair of Lie algebroids is a pair of Lie algebroids $(\mathcal{A}, \mathcal{B})$ together with two representations $\rho_{\mathcal{A}}: \mathcal{A} \longrightarrow \mathfrak{D}(\mathcal{B})$ and $\rho_{\mathcal{B}}: \mathcal{B} \longrightarrow$ $\mathfrak{D}(\mathcal{A})$ such that some compatibility conditions are satisfied.

For all $x \in \Gamma(\mathcal{A})$, the Lie derivation $\mathcal{L}_{x}: \Gamma\left(\mathcal{A}^{*}\right) \longrightarrow \Gamma\left(\mathcal{A}^{*}\right)$ of the Lie algebroid $\mathcal{A}$ is given by

$$
\left\langle\mathcal{L}_{x} \xi, y\right\rangle=a_{\mathcal{A}}(x)\langle\xi, y\rangle-\left\langle\xi,[x, y]_{\mathcal{A}}\right\rangle, \quad \forall y \in \Gamma(\mathcal{A}), \xi \in \Gamma\left(\mathcal{A}^{*}\right) .
$$

A Lie algebroid $\left(\mathcal{A},[\cdot, \cdot]_{\mathcal{A}}, a_{\mathcal{A}}\right)$ naturally represents on the trivial line bundle $E=M \times \mathbb{R}$ via the anchor map $a_{\mathcal{A}}: \mathcal{A} \longrightarrow T M$. The corresponding coboundary operator $\mathrm{d}: \Gamma\left(\wedge^{k} \mathcal{A}^{*}\right) \longrightarrow \Gamma\left(\wedge^{k+1} \mathcal{A}^{*}\right)$ is given by

$$
\begin{aligned}
\mathrm{d} \varpi\left(x_{1}, \cdots, x_{k+1}\right)= & \sum_{i=1}^{k+1}(-1)^{i+1} a_{\mathcal{A}}\left(x_{i}\right) \varpi\left(x_{1} \cdots, \widehat{x_{i}}, \cdots, x_{k+1}\right) \\
& +\sum_{i<j}(-1)^{i+j} \varpi\left(\left[x_{i}, x_{j}\right]_{\mathcal{A}}, x_{1} \cdots, \widehat{x_{i}}, \cdots, \widehat{x_{j}}, \cdots, x_{k+1}\right) .
\end{aligned}
$$


In particular, a 2-form $\varpi \in \Gamma\left(\wedge^{2} \mathcal{A}^{*}\right)$ is a 2-cocycle if $\mathrm{d} \varpi=0$, i.e.

$$
a_{\mathcal{A}}(x) \varpi(y, z)-a_{\mathcal{A}}(y) \varpi(x, z)+a_{\mathcal{A}}(z) \varpi(x, y)-\varpi\left([x, y]_{\mathcal{A}}, z\right)+\varpi\left([x, z]_{\mathcal{A}}, y\right)-\varpi\left([y, z]_{\mathcal{A}}, x\right)=0 .
$$

A symplectic Lie algebroid is a Lie algebroid together with a nondegenerate closed 2-form. A subalgebroid of a symplectic Lie algebroid $\left(\mathcal{A},[\cdot, \cdot]_{\mathcal{A}}, a_{\mathcal{A}}, \varpi\right)$ is called Lagrangian if it is maximal isotropic with respect to the skew-symmetric bilinear form $\varpi$.

\section{Left-symmetric algebroids}

Definition 2.2. A left-symmetric algebra is a pair $(\mathfrak{g}, \cdot \mathfrak{g})$, where $\mathfrak{g}$ is a vector space, and $\cdot \mathfrak{g}: \mathfrak{g} \otimes \mathfrak{g} \longrightarrow \mathfrak{g}$ is a bilinear multiplication satisfying that for all $x, y, z \in \mathfrak{g}$, the associator

$$
(x, y, z) \triangleq x \cdot \mathfrak{g}(y \cdot \mathfrak{g} z)-(x \cdot \mathfrak{g} y) \cdot \mathfrak{g} z
$$

is symmetric in $x, y$, i.e.

$(x, y, z)=(y, x, z)$, or equivalently, $x \cdot \mathfrak{g}(y \cdot \mathfrak{g} z)-(x \cdot \mathfrak{g} y) \cdot_{\mathfrak{g}} z=y \cdot \mathfrak{g}(x \cdot \mathfrak{g} z)-(y \cdot \mathfrak{g} x) \cdot_{\mathfrak{g}} z$.

A left-symmetric algebroid is also called a Koszul-Vinberg algebroid in [22].

Definition 2.3. 12, 22, $A$ left-symmetric algebroid structure on a vector bundle $A \longrightarrow M$ is a pair that consists of a left-symmetric algebra structure $\cdot_{A}$ on the section space $\Gamma(A)$ and a vector bundle morphism $a_{A}: A \longrightarrow T M$, called the anchor, such that for all $f \in C^{\infty}(M)$ and $x, y \in \Gamma(A)$, the following conditions are satisfied:

(i) $x \cdot{ }_{A}(f y)=f\left(x \cdot{ }_{A} y\right)+a_{A}(x)(f) y$,

(ii) $(f x) \cdot{ }_{A} y=f\left(x \cdot{ }_{A} y\right)$.

We usually denote a left-symmetric algebroid by $\left(A, \cdot_{A}, a_{A}\right)$. Any left-symmetric algebra is a left-symmetric algebroid over a point.

Example 2.4. Let $M$ be a differential manifold with a flat torsion free connection $\nabla$. Then $(T M, \nabla$, id $)$ is a left-symmetric algebroid whose sub-adjacent Lie algebroid is exactly the tangent Lie algebroid. We denote this left-symmetric algebroid by $T_{\nabla} M$, which will be frequently used below.

For any $x \in \Gamma(A)$, we define $L_{x}: \Gamma(A) \longrightarrow \Gamma(A)$ and $R_{x}: \Gamma(A) \longrightarrow \Gamma(A)$ by

$$
L_{x} y=x \cdot{ }_{A} y, \quad R_{x} y=y \cdot{ }_{A} x .
$$

Condition (i) in the above definition means that $L_{x} \in \mathfrak{D}(A)$. Condition (ii) means that the map $x \longmapsto L_{x}$ is $C^{\infty}(M)$-linear. Thus, $L: A \longrightarrow \mathfrak{D}(A)$ is a bundle map.

Proposition 2.5. 12] Let $\left(A, \cdot_{A}, a_{A}\right)$ be a left-symmetric algebroid. Define a skew-symmetric bilinear bracket operation $[\cdot, \cdot]_{A}$ on $\Gamma(A)$ by

$$
[x, y]_{A}=x \cdot{ }_{A} y-y \cdot{ }_{A} x, \quad \forall x, y \in \Gamma(A) .
$$

Then, $\left(A,[\cdot, \cdot]_{A}, a_{A}\right)$ is a Lie algebroid, and denoted by $A^{c}$, called the sub-adjacent Lie algebroid of $\left(A, \cdot_{A}, a_{A}\right)$. Furthermore, $L: A \longrightarrow \mathfrak{D}(A)$ gives a representation of the Lie algebroid $A^{c}$. 
Theorem 2.6. [12] Let $\left(A, \cdot A_{A}, a_{A}\right)$ be a left-symmetric algebroid. Then $\left(A^{c} \ltimes_{L^{*}} A^{*},[\cdot, \cdot]_{S}, \rho, \omega\right)$ is a symplectic Lie algebroid, where $A^{c} \ltimes_{L^{*}} A^{*}$ is the semidirect product of $A^{c}$ and $A^{*}$ in which $L^{*}$ is the dual representation of $L$. More precisely, the Lie bracket $[\cdot, \cdot]_{S}$ and the anchor $\rho$ are given by

$$
[x+\xi, y+\eta]_{S}=[x, y]_{A}+L_{x}^{*} \eta-L_{y}^{*} \xi,
$$

and $\rho(x+\xi)=a_{A}(x)$ respectively. Furthermore, the symplectic form $\omega$ is given by

$$
\omega(x+\xi, y+\eta)=\langle\xi, y\rangle-\langle\eta, x\rangle, \quad \forall x, y \in \Gamma(A), \xi, \eta \in \Gamma\left(A^{*}\right) .
$$

Let $\left(A, \cdot_{A}, a_{A}\right)$ be a left-symmetric algebroid and $E$ a vector bundle. A representation of $A$ on $E$ consists of a pair $(\rho, \mu)$, where $\rho: A \longrightarrow \mathfrak{D}(E)$ is a representation of $A^{c}$ on $E$ and $\mu: A \longrightarrow \operatorname{End}(E)$ is a bundle map, such that for all $x, y \in \Gamma(A), e \in \Gamma(E)$, we have

$$
\rho(x) \mu(y) e-\mu(y) \rho(x) e=\mu(x \cdot A y) e-\mu(y) \mu(x) e .
$$

Denote a representation by $(E ; \rho, \mu)$.

Let us recall the cohomology complex with the coefficients in the trivial representation, i.e. $\rho=a_{A}$ and $\mu=0$. See [5, 12] for general theory of cohomologies of right-symmetric algebras and left-symmetric algebroids respectively. The set of $(n+1)$-cochains is given by

$$
C^{n+1}(A)=\Gamma\left(\wedge^{n} A^{*} \otimes A^{*}\right), n \geq 0 .
$$

For all $\varphi \in C^{n}(A)$ and $x_{i} \in \Gamma(A), i=1, \cdots, n+1$, the corresponding coboundary operator $\delta$ is given by

$$
\begin{aligned}
\delta \varphi\left(x_{1}, \cdots, x_{n+1}\right)= & \sum_{i=1}^{n}(-1)^{i+1} a_{A}\left(x_{i}\right) \varphi\left(x_{1}, \cdots, \hat{x_{i}}, \cdots, x_{n+1}\right) \\
& -\sum_{i=1}^{n}(-1)^{i+1} \varphi\left(x_{1}, \cdots, \hat{x}_{i}, \cdots, x_{n}, x_{i} \cdot A_{A} x_{n+1}\right) \\
& +\sum_{1 \leq i<j \leq n}(-1)^{i+j} \varphi\left(\left[x_{i}, x_{j}\right]_{A}, x_{1}, \cdots, \hat{x}_{i}, \cdots, \hat{x}_{j}, \cdots, x_{n+1}\right) .
\end{aligned}
$$

\section{Pre-symplectic algebroids}

Here we recall the notion of pre-symplectic algebroids and the relation with symplectic Lie algebroids. See [13] for more details.

Definition 2.7. A pre-symplectic algebroid is a vector bundle $E \rightarrow M$ equipped with a nondegenerate skew-symmetric bilinear form $(\cdot, \cdot)_{-}$, a multiplication $\star: \Gamma(E) \times \Gamma(E) \longrightarrow \Gamma(E)$, and a bundle map $\rho: E \rightarrow T M$, such that for all $e, e_{1}, e_{2}, e_{3} \in \Gamma(E), f \in C^{\infty}(M)$, the following conditions are satisfied:

(i) $\left(e_{1}, e_{2}, e_{3}\right)-\left(e_{2}, e_{1}, e_{3}\right)=\frac{1}{6} D T\left(e_{1}, e_{2}, e_{3}\right)$;

(ii) $\rho\left(e_{1}\right)\left(e_{2}, e_{3}\right)_{-}=\left(e_{1} \star e_{2}-\frac{1}{2} D\left(e_{1}, e_{2}\right)_{-}, e_{3}\right)_{-}+\left(e_{2},\left[e_{1}, e_{3}\right]_{E}\right)_{-}$,

where $\left(e_{1}, e_{2}, e_{3}\right)$ is the associator for the multiplication $\star$ given by (3), $T: \Gamma(E) \times \Gamma(E) \times \Gamma(E) \longrightarrow$ $C^{\infty}(M)$ is defined by

$$
T\left(e_{1}, e_{2}, e_{3}\right)=\left(e_{1} \star e_{2}, e_{3}\right)_{-}+\left(e_{1}, e_{2} \star e_{3}\right)_{-}-\left(e_{2} \star e_{1}, e_{3}\right)_{-}-\left(e_{2}, e_{1} \star e_{3}\right)_{-},
$$


$D: C^{\infty}(M) \longrightarrow \Gamma(E)$ is defined by

$$
(D f, e)_{-}=\rho(e)(f),
$$

and the bracket $[\cdot, \cdot]_{E}: \wedge^{2} \Gamma(E) \longrightarrow \Gamma(E)$ is defined by

$$
\left[e_{1}, e_{2}\right]_{E}=e_{1} \star e_{2}-e_{2} \star e_{1} .
$$

We denote a pre-symplectic algebroid by $\left(E, \star, \rho,(\cdot, \cdot)_{-}\right)$.

Theorem 2.8. Let $\left(E, \star, \rho,(\cdot, \cdot)_{-}\right)$be a pre-symplectic algebroid. Then $\left(E,[\cdot, \cdot]_{E}, \rho, \omega=(\cdot, \cdot)_{-}\right)$is a symplectic Lie algebroid.

Given a symplectic Lie algebroid $\left(E,[\cdot, \cdot]_{E}, \rho, \omega\right)$, define a multiplication $\star: \Gamma(E) \times \Gamma(E) \longrightarrow$ $\Gamma(E)$ by

$$
e_{1} \star e_{2}=\omega^{\sharp}-1\left(\mathcal{L}_{e_{1}} \omega^{\sharp}\left(e_{2}\right)+\frac{1}{2} \mathrm{~d}\left(\omega\left(e_{1}, e_{2}\right)\right)\right) \quad \forall e_{1}, e_{2} \in \Gamma(E) .
$$

Theorem 2.9. Let $\left(E,[\cdot, \cdot]_{E}, \rho, \omega\right)$ be a symplectic Lie algebroid. Then $\left(E, \star, \rho,(\cdot, \cdot)_{-}=\omega\right)$ is a pre-symplectic algebroid, and satisfies

$$
\left[e_{1}, e_{2}\right]_{E}=e_{1} \star e_{2}-e_{2} \star e_{1}, \quad \forall e_{1}, e_{2} \in \Gamma(E),
$$

where the multiplication $\star$ is given by (11).

Example 2.10. Let $\left(A,{ }_{A}, a_{A}\right)$ be a left-symmetric algebroid and $\left(A^{c} \ltimes_{L^{*}} A^{*}, \omega\right)$ the corresponding symplectic Lie algebroid, where $\omega$ is given by (5). Then the corresponding pre-symplectic algebroid structure is given by

$$
(x+\xi) \star(y+\eta)=x \cdot A y+\mathcal{L}_{x} \eta-R_{y} \xi-\frac{1}{2} \mathrm{~d}(x+\xi, y+\eta)_{+},
$$

where $\mathcal{L}$ is given by (11), $R$ is given by (23), and $(\cdot, \cdot)_{+}$is the nondegenerate symmetric bilinear form on $A \oplus A^{*}$ given by

$$
(x+\xi, y+\eta)_{+}=\langle\xi, y\rangle+\langle\eta, x\rangle .
$$

Definition 2.11. Let $\left(E, \star, \rho,(\cdot, \cdot)_{-}\right)$be a pre-symplectic algebroid. A subbundle $F$ of $E$ is called isotropic if it is isotropic under the skew-symmetric bilinear form $(\cdot, \cdot)_{-}$. It is called integrable if $\Gamma(F)$ is closed under the operation $\star$. A Dirac structure is a subbundle $F$ which is maximal isotropic and integrable.

The following proposition is obvious.

Proposition 2.12. Let $F$ be a Dirac structure of a pre-symplectic algebroid $\left(E, \star, \rho,(\cdot, \cdot)_{-}\right)$. Then $\left(F,\left.\star\right|_{F},\left.\rho\right|_{F}\right)$ is a left-symmetric algebroid.

\section{Differential calculus on left-symmetric algebroids}

In this section, we develop the differential calculus on left-symmetric algebroids, which is the fundamental tool in the following study. Let $\left(A, \cdot_{A}, a_{A}\right)$ be a left-symmetric algebroid. For all $x \in \Gamma(A)$, define the Lie derivative $\mathfrak{L}_{x}: \Gamma\left(\wedge^{n} A \otimes A\right) \longrightarrow \Gamma\left(\wedge^{n} A \otimes A\right)$ by

$\mathfrak{L}_{x}\left(y_{1} \wedge \cdots \wedge y_{n} \otimes y_{n+1}\right)=\sum_{i=1}^{n} y_{1} \wedge \cdots \wedge x \cdot A y_{i} \wedge \cdots \wedge y_{n} \otimes y_{n+1}+y_{1} \wedge \cdots \wedge y_{n} \otimes\left[x, y_{n+1}\right]_{A}$, 
where $y_{1}, \cdots, y_{n+1} \in \Gamma(A)$. Define the right multiplication $R_{x}: \Gamma\left(\wedge^{n} A \otimes A\right) \longrightarrow \Gamma\left(\wedge^{n} A \otimes A\right)$ by

$$
R_{x}\left(y_{1} \wedge \cdots \wedge y_{n} \otimes y_{n+1}\right)=-\left(\sum_{i=1}^{n} y_{1} \wedge \cdots y_{i} \cdot A x \cdots \wedge y_{n} \otimes y_{n+1}\right)+y_{1} \wedge \cdots \wedge y_{n} \otimes y_{n+1} \cdot A x
$$

Remark 3.1. For all $x, y \in \Gamma(A)$, we have $\mathfrak{L}_{x} y=[x, y]_{A}$. Thus, $\mathfrak{L}_{x}$ is not a straightforward generalization of the left multiplication $L_{x}: \Gamma(A) \longrightarrow \Gamma(A)$ given by (4). This is why we use different notations. However, $R_{x}: \Gamma\left(\wedge^{n} A \otimes A\right) \longrightarrow \Gamma\left(\wedge^{n} A \otimes A\right)$ is a straightforward generalization of the right multiplication $R_{x}: \Gamma(A) \longrightarrow \Gamma(A)$ given by (4). Therefore, we use the same notations which will not cause confusion.

For all $\xi \in \Gamma\left(A^{*}\right)$, the left contraction and right contraction, which we denote by $\left.\xi\right\lrcorner$ : $\Gamma\left(\wedge^{n} A \otimes A\right) \longrightarrow \Gamma\left(\wedge^{n-1} A \otimes A\right)$ and $\left\llcorner\xi: \Gamma\left(\wedge^{n} A \otimes A\right) \longrightarrow \Gamma\left(\wedge^{n} A\right)\right.$ respectively, are defined by

$$
\begin{aligned}
& (\xi\lrcorner \varphi)\left(\eta_{1}, \eta_{2}, \cdots, \eta_{n}\right)=\varphi\left(\xi, \eta_{1}, \eta_{2}, \cdots, \eta_{n}\right) ; \\
& \left(\varphi\llcorner\xi)\left(\eta_{1}, \eta_{2}, \cdots, \eta_{n}\right)=\varphi\left(\eta_{1}, \eta_{2}, \cdots, \eta_{n}, \xi\right),\right.
\end{aligned}
$$

where $\varphi \in \Gamma\left(\wedge^{n} A \otimes A\right), \quad n \geq 1$ and $\eta_{1}, \eta_{2}, \cdots, \eta_{n} \in \Gamma\left(A^{*}\right)$.

The Lie derivative $\mathfrak{L}_{x}$ has the following properties.

Proposition 3.2. For all $x, y \in \Gamma(A), f \in C^{\infty}(M), X \in \Gamma\left(\wedge^{n} A \otimes A\right), n \geq 0$, we have

$$
\begin{aligned}
\mathfrak{L}_{[x, y]_{A}} & =\left[\mathfrak{L}_{x}, \mathfrak{L}_{y}\right] ; \\
\mathfrak{L}_{x} f X & =f \mathfrak{L}_{x} X+a_{A}(x)(f) X ; \\
\mathfrak{L}_{f x} X & =f \mathfrak{L}_{x} X-X\left\llcorner_{\mathrm{d} f} \otimes x .\right.
\end{aligned}
$$

Proof. We only prove (21). Others can be proved similarly. For all $x, y_{1}, y_{2}, \cdots, y_{n+1} \in \Gamma(A), f \in$ $C^{\infty}(M)$, without loss of generality we can assume that $X=y_{1} \wedge \cdots \wedge y_{n} \otimes y_{n+1}$, then we have

$$
\begin{aligned}
& \mathfrak{L}_{f x} y_{1} \wedge \cdots \wedge y_{n} \otimes y_{n+1} \\
= & \sum_{i=1}^{n} y_{1} \wedge \cdots \wedge(f x) \cdot{ }_{A} y_{i} \wedge \cdots \wedge y_{n} \otimes y_{n+1}+y_{1} \wedge \cdots \wedge y_{n} \otimes\left[f x, y_{n+1}\right]_{A} \\
= & f \sum_{i=1}^{n} y_{1} \wedge \cdots \wedge x \cdot{ }_{A} y_{i} \wedge \cdots \wedge y_{n} \otimes y_{n+1}+f y_{1} \wedge \cdots \wedge y_{n} \otimes\left[x, y_{n+1}\right]_{A} \\
& -y_{1} \wedge \cdots \wedge y_{n} \otimes a_{A}\left(y_{n+1}\right)(f) x \\
= & f \mathfrak{L}_{x} y_{1} \wedge \cdots \wedge y_{n} \otimes y_{n+1}-\left(y_{1} \wedge \cdots \wedge y_{n} \otimes y_{n+1}\right)\left\llcorner_{\mathrm{d} f} \otimes x .\right.
\end{aligned}
$$

The proof is finished.

For all $x \in \Gamma(A)$, the Lie derivative $\mathfrak{L}_{x}: \Gamma\left(\wedge^{n} A^{*} \otimes A^{*}\right) \longrightarrow \Gamma\left(\wedge^{n} A^{*} \otimes A^{*}\right)$ and the right multiplication $R_{x}: \Gamma\left(\wedge^{n} A^{*} \otimes A^{*}\right) \longrightarrow \Gamma\left(\wedge^{n} A^{*} \otimes A^{*}\right)$ are defined respectively by 2

$$
\begin{aligned}
\left\langle\mathfrak{L}_{x} \varphi, X\right\rangle & =a_{A}(x)\langle\varphi, X\rangle-\left\langle\varphi, \mathfrak{L}_{x} X\right\rangle ; \\
\left\langle R_{x} \varphi, X\right\rangle & =-\left\langle\varphi, R_{x} X\right\rangle .
\end{aligned}
$$

where $\varphi \in \Gamma\left(\wedge^{n} A^{*} \otimes A^{*}\right), \quad n \geq 1$ and $X \in \Gamma\left(\wedge^{n} A \otimes A\right)$.

These operators satisfy the following equalities which are repeatedly used below.

\footnotetext{
${ }^{2}$ Here we use the same notations as before and this will not bring confusion since it depends on what it acts.
} 
Proposition 3.3. For all $\varphi \in \Gamma\left(\wedge^{n} A^{*} \otimes A^{*}\right), n \geq 1, x, y \in \Gamma(A), \xi \in \Gamma\left(A^{*}\right)$, we have

$$
\begin{aligned}
\delta(f \xi) & =f \delta(\xi)+\mathrm{d} f \otimes \xi ; \\
\mathfrak{L}_{[x, y]_{A}} & =\left[\mathfrak{L}_{x}, \mathfrak{L}_{y}\right] \\
\left.\left(x \cdot{ }_{A} y\right)\right\lrcorner \varphi & \left.\left.=\mathfrak{L}_{x}\left({ }_{y}\right\lrcorner \varphi\right)-{ }_{y}\right\lrcorner\left(\mathfrak{L}_{x} \varphi\right) ; \\
\mathfrak{L}_{x} \varphi & \left.\left.=\delta\left({ }_{x}\right\lrcorner \varphi\right)+\left({ }_{x}\right\lrcorner\right) \delta \varphi-R_{x} \varphi ; \\
\mathfrak{L}_{x} f \varphi & =f \mathfrak{L}_{x} \varphi+a_{A}(x)(f) \varphi ; \\
\mathfrak{L}_{f x} \varphi & =f \mathfrak{L}_{x} \varphi+\varphi\left\llcorner_{x} \otimes \mathrm{d} f ;\right. \\
R_{x} f \xi & =f R_{x} \xi ; \\
R_{f x} \xi & =f R_{x} \xi-\langle x, \xi\rangle \mathrm{d} f .
\end{aligned}
$$

Proof. We only give the proof of (26) and (27). Others can be proved similarly. For all $\varphi \in$ $\Gamma\left(\wedge^{n} A^{*} \otimes A^{*}\right), \quad n \geq 1$ and $x, y, y_{1}, y_{2}, \cdots, y_{n} \in \Gamma(A)$, we have

$$
\begin{aligned}
& \left.\mathfrak{L}_{x}\left({ }_{y}\right\lrcorner \varphi\right)\left(y_{1}, \cdots, y_{n}\right) \\
= & \left.\left.\left.a_{A}(x)_{y}\right\lrcorner \varphi\left(y_{1}, \cdots, y_{n}\right)-{ }_{y}\right\lrcorner \varphi\left(y_{1}, \cdots,\left[x, y_{n}\right]_{A}\right)-\sum_{i=1}^{n-1} y_{y}\right\lrcorner \varphi\left(y_{1}, \cdots, x \cdot{ }_{A} y_{i}, \cdots, y_{n}\right) \\
= & a_{A}(x) \varphi\left(y, y_{1}, \cdots, y_{n}\right)-\varphi\left(y, y_{1}, \cdots,\left[x, y_{n}\right]_{A}\right)-\sum_{i=1}^{n-1} \varphi\left(y, y_{1}, \cdots, x \cdot \cdot_{A} y_{i}, \cdots, y_{n}\right)
\end{aligned}
$$

and

$$
\begin{aligned}
& y\lrcorner\left(\mathfrak{L}_{x} \varphi\right)\left(y_{1}, \cdots, y_{n}\right)=\mathfrak{L}_{x} \varphi\left(y, y_{1}, \cdots, y_{n}\right) \\
= & a_{A}(x) \varphi\left(y, y_{1}, \cdots, y_{n}\right)-\varphi\left(y, y_{1}, \cdots,\left[x, y_{n}\right]_{A}\right)-\sum_{i=1}^{n-1} \varphi\left(y, y_{1}, \cdots, x \cdot{ }_{A} y_{i}, \cdots, y_{n}\right) \\
& -\varphi\left(x \cdot A y, y_{1}, \cdots, y_{n}\right) .
\end{aligned}
$$

Therefore, we have

$$
\left.\left.\mathfrak{L}_{x}\left({ }_{y}\right\lrcorner \varphi\right)\left(y_{1}, \cdots, y_{n}\right){ }_{y}\right\lrcorner\left(\mathfrak{L}_{x} \varphi\right)\left(y_{1}, \cdots, y_{n}\right)=\varphi\left(x \cdot{ }_{A} y, y_{1}, \cdots, y_{n}\right),
$$

which implies that (26) holds.

Next we prove that (27) holds. On one hand, for all $\varphi \in \Gamma\left(\wedge^{n} A^{*} \otimes A^{*}\right), n \geq 1$ and $x, y_{1}, \cdots, y_{n+1} \in \Gamma(A)$, we have

$$
\begin{aligned}
& \left.\left({ }_{x}\right\lrcorner\right) \delta \varphi\left(y_{1}, \cdots, y_{n+1}\right)=\delta \varphi\left(x, y_{1}, \cdots, y_{n+1}\right) \\
= & a_{A}(x) \varphi\left(y_{1}, \cdots, y_{n+1}\right)+\sum_{i=1}^{n}(-1)^{i} a_{A}\left(y_{i}\right) \varphi\left(x, y_{1}, \cdots, \hat{y_{i}}, \cdots, y_{n+1}\right) \\
& -\varphi\left(y_{1}, \cdots, y_{n}, x \cdot \cdot_{A} y_{n+1}\right)-\sum_{i=1}^{n}(-1)^{i} \varphi\left(x, y_{1}, \cdots, \hat{y}_{i}, \cdots, y_{n}, y_{i} \cdot{ }_{A} y_{n+1}\right) \\
& +\sum_{i=1}^{n}(-1)^{i} \varphi\left(\left[x, y_{i}\right]_{A}, y_{1}, \cdots, \hat{y_{i}}, \cdots, y_{n+1}\right) \\
& +\sum_{1 \leq i<j \leq n}(-1)^{i+j} \varphi\left(\left[y_{i}, y_{j}\right]_{A}, x, y_{1}, \cdots, \hat{y}_{i}, \cdots, \hat{y_{j}}, \cdots, y_{n+1}\right)
\end{aligned}
$$


and

$$
\begin{aligned}
\left.\delta\left({ }_{x}\right\lrcorner \varphi\right)\left(y_{1}, \cdots, y_{n+1}\right)= & \sum_{i=1}^{n}(-1)^{i+1} a_{A}\left(y_{i}\right) \varphi\left(x, y_{1}, \cdots, \hat{y_{i}}, \cdots, y_{n+1}\right) \\
& -\sum_{i=1}^{n}(-1)^{i+1} \varphi\left(x, y_{1}, \cdots, \hat{y_{i}}, \cdots, y_{n}, y_{i} \cdot{ }_{A} y_{n+1}\right) \\
& +\sum_{1 \leq i<j \leq n}(-1)^{i+j} \varphi\left(x,\left[y_{i}, y_{j}\right]_{A}, y_{1}, \cdots, \hat{y}_{i}, \cdots, \hat{y}_{j}, \cdots, y_{n+1}\right) .
\end{aligned}
$$

On the other hand, by (22) and (23), we have

$$
\begin{aligned}
& \left(\mathfrak{L}_{x} \varphi+R_{x} \varphi\right)\left(y_{1}, \cdots, y_{n+1}\right) \\
= & a_{A}(x) \varphi\left(y_{1}, \cdots, y_{n+1}\right)-\varphi\left(y_{1}, \cdots, y_{n}, x \cdot_{A} y_{n+1}\right) \\
& +\sum_{i=1}^{n}(-1)^{i} \varphi\left(\left[x, y_{i}\right]_{A}, y_{1}, \cdots, \hat{y}_{i}, \cdots, y_{n+1}\right) .
\end{aligned}
$$

Thus, (27) holds.

\section{Left-symmetric bialgebroids}

In this section, we introduce the concept of a left-symmetric bialgebroid and study its properties. We construct a left-symmetric algebroid using a symmetric tensor satisfying a condition, which can be viewed as a generalization of the S-equation introduced in 1 for left-symmetric bialgebras. In particular, we construct a left-symmetric bialgebroid using a pseudo-Hessian manifold.

Definition 4.1. Let $\left(A, \cdot{ }_{A}, a_{A}\right)$ and $\left(A^{*}, \cdot_{A^{*}}, a_{A^{*}}\right)$ be two left-symmetric algebroids. Then $\left(A, A^{*}\right)$ is a left-symmetric bialgebroid if for all $x, y \in \Gamma(A), \xi, \eta \in \Gamma\left(A^{*}\right)$, the following equalities hold:

$$
\begin{aligned}
\delta[\xi, \eta]_{A^{*}} & =\mathfrak{L}_{\xi} \delta \eta-\mathfrak{L}_{\eta} \delta \xi ; \\
\delta_{*}[x, y]_{A} & =\mathfrak{L}_{x} \delta_{*} y-\mathfrak{L}_{y} \delta_{*} x,
\end{aligned}
$$

where $\delta$ and $\delta_{*}$ are coboundary operators of left-symmetric algebroids $A$ and $A^{*}$ respectively and $\mathfrak{L}$ is the Lie derivative associated to a left-symmetric algebroid given by (15).

Remark 4.2. By (15), it is not hard to see that a left-symmetric bialgebroid reduces to a leftsymmetric bialgebra when the base manifold is a point. Thus, a left-symmetric bialgebroid can be viewed as a geometric generalization of a left-symmetric bialgebra. See [1] for more details about left-symmetric bialgebras.

Lemma 4.3. Let $\left(A, A^{*}\right)$ be a left-symmetric bialgebroid. For all $x \in \Gamma(A), \xi \in \Gamma\left(A^{*}\right), f \in$ $C^{\infty}(M)$, we have

$$
\begin{aligned}
x \cdot A & \mathrm{~d}_{*} f=\mathrm{d}_{*}\left(x\left\llcorner_{\mathrm{d} f}\right)-\left(\delta_{*} x\right)\left\llcorner_{\mathrm{d} f},\right.\right. \\
\xi \cdot A^{*} \mathrm{~d} f & =\mathrm{d}\left(x\left\llcorner_{\mathrm{d}_{*} f}\right)-(\delta \xi)\left\llcorner_{\mathrm{d}_{*} f} .\right.\right.
\end{aligned}
$$


Proof. By (201), (21) and (33), we have

$$
\begin{aligned}
\delta_{*}[x, f y]_{A} & =\mathfrak{L}_{x} \delta_{*} f y-\mathfrak{L}_{f y} \delta_{*} x \\
& =\mathfrak{L}_{x}\left(f \delta_{*} y+\mathrm{d}_{*} f \otimes y\right)-\left(f \mathfrak{L}_{y} \delta_{*} x-\left(\delta_{*} x\right)\left\llcorner_{\mathrm{d} f} \otimes y\right)\right. \\
& =f \mathfrak{L}_{x} \delta_{*} y+a_{A}(x)(f) \delta_{*} y+\mathfrak{L}_{x}\left(\mathrm{~d}_{*} f \otimes y\right)-f \mathfrak{L}_{y} \delta_{*} x+\left(\delta_{*} x\right)_{\mathrm{d} f} \otimes y \\
& =f \delta_{*}[x, y]_{A}+a_{A}(x)(f) \delta_{*} y+\left(x \cdot \cdot_{A} \mathrm{~d}_{*} f\right) \otimes y+\mathrm{d}_{*} f \otimes[x, y]_{A}+\left(\delta_{*} x\right)\left\llcorner_{\mathrm{d} f} \otimes y .\right.
\end{aligned}
$$

On the other hand, we have

$$
\begin{aligned}
\delta_{*}[x, f y]_{A} & =\delta_{*}\left(f[x, y]_{A}+a_{A}(x)(f) y\right) \\
& =f \delta_{*}[x, y]_{A}+\mathrm{d}_{*} f \otimes[x, y]_{A}+a_{A}(x)(f) \delta_{*} y+\mathrm{d}_{*} a_{A}(x)(f) \otimes y .
\end{aligned}
$$

Thus, we have

$$
x \cdot{ }_{A} \mathrm{~d}_{*} f+\left(\delta_{*} x\right)\left\llcorner_{\mathrm{d} f}=\mathrm{d}_{*} a_{A}(x)(f)=\mathrm{d}_{*}\left(x\left\llcorner_{\mathrm{d} f}\right),\right.\right.
$$

which implies that (34) holds. (35) can be proved similarly.

Recall that $R_{x}: A^{*} \longrightarrow A^{*}$ and $R_{\xi}: A \longrightarrow A$ are defined by

$$
\left\langle R_{x} \xi, y\right\rangle=-\left\langle\xi, y \cdot A_{A} x\right\rangle, \quad\left\langle R_{\xi} x, \eta\right\rangle=-\left\langle x, \eta \cdot A^{*} \xi\right\rangle .
$$

Corollary 4.4. Let $\left(A, A^{*}\right)$ be a left-symmetric bialgebroid. For all $x \in \Gamma(A), \xi \in \Gamma\left(A^{*}\right), f \in$ $C^{\infty}(M)$, we have

$$
\begin{aligned}
x \cdot{ }_{A} \mathrm{~d}_{*} f & =-R_{\mathrm{d} f} x, \\
\xi \cdot A^{*} \mathrm{~d} f & =-R_{\mathrm{d}_{*} f} \xi .
\end{aligned}
$$

Proof. By (34), we have

$$
\begin{aligned}
& \left\langle\mathrm { d } _ { * } \left( x\llcorner\mathrm{~d} f)-\left(\delta_{*}(x)\left\llcorner_{\mathrm{d} f}, \xi\right\rangle=\left\langle\mathrm{d}_{*} a_{A}(x)(f), \xi\right\rangle-\left\langle\delta_{*}(x), \xi \otimes \mathrm{d} f\right\rangle\right.\right.\right. \\
& =a_{A^{*}}(\xi) a_{A}(x)(f)-a_{A^{*}}(\xi) a_{A}(x)(f)+\left\langle x, \xi \cdot A^{*} \mathrm{~d} f\right\rangle \\
& =\left\langle-R_{\mathrm{d} f} x, \xi\right\rangle \text {, }
\end{aligned}
$$

which implies that (36) holds. (37) can be proved similarly.

Corollary 4.5. Let $\left(A, A^{*}\right)$ be a left-symmetric bialgebroid. For all $x \in \Gamma(A), \xi \in \Gamma\left(A^{*}\right)$, we have

$$
\left[a_{A}(x), a_{A^{*}}(\xi)\right]=a_{A^{*}}\left(L_{x}^{*} \xi\right)-a_{A}\left(L_{\xi}^{*} x\right) .
$$

Proof. For all $f \in C^{\infty}(M)$, by (36), we have

$$
\begin{aligned}
\left\langle a_{A^{*}}\left(L_{x}^{*} \xi\right)-a_{A}\left(L_{\xi}^{*} x\right), d f\right\rangle & =\left\langle\mathrm{d}_{*} f, L_{x}^{*} \xi\right\rangle-\left\langle\mathrm{d} f, L_{\xi}^{*} x\right\rangle \\
& =a_{A}(x)\left\langle\mathrm{d}_{*} f, \xi\right\rangle-\left\langle x \cdot{ }_{A} \mathrm{~d}_{*} f, \xi\right\rangle-a_{A^{*}}(\xi)\langle\mathrm{d} f, x\rangle+\left\langle\xi \cdot A^{*} \mathrm{~d} f, x\right\rangle \\
& =a_{A}(x)\left\langle\mathrm{d}_{*} f, \xi\right\rangle-a_{A^{*}}(\xi)\langle\mathrm{d} f, x\rangle+\left\langle R_{\mathrm{d} f} x, \xi\right\rangle+\left\langle\xi \cdot A^{*} \mathrm{~d} f, x\right\rangle \\
& =\left\langle\left[a_{A}(x), a_{A^{*}}(\xi)\right], d f\right\rangle,
\end{aligned}
$$

which implies that (38) holds.

Let $\left(A,{ }_{A}, a_{A}\right)$ be a left-symmetric algebroid. Define

$$
\operatorname{Sym}^{2}(A)=\left\{H \in A \otimes A \mid H(\xi, \eta)=H(\eta, \xi), \quad \forall \xi, \eta \in \Gamma\left(A^{*}\right)\right\} .
$$


For any $H \in \operatorname{Sym}^{2}(A)$, the bundle map $H^{\sharp}: A^{*} \longrightarrow A$ is given by $H^{\sharp}(\xi)(\eta)=H(\xi, \eta)$. We introduce $\llbracket H, H \rrbracket \in \wedge^{2} A \otimes A$ as follows:

$$
\begin{aligned}
\llbracket H, H \rrbracket\left(\xi_{1}, \xi_{2}, \xi_{3}\right)= & a_{A}\left(H^{\sharp}\left(\xi_{1}\right)\right)\left\langle H^{\sharp}\left(\xi_{2}\right), \xi_{3}\right\rangle-a_{A}\left(H^{\sharp}\left(\xi_{2}\right)\right)\left\langle H^{\sharp}\left(\xi_{1}\right), \xi_{3}\right\rangle+\left\langle\xi_{1}, H^{\sharp}\left(\xi_{2}\right) \cdot{ }_{A} H^{\sharp}\left(\xi_{3}\right)\right\rangle \\
& -\left\langle\xi_{2}, H^{\sharp}\left(\xi_{1}\right) \cdot{ }_{A} H^{\sharp}\left(\xi_{3}\right)\right\rangle-\left\langle\xi_{3},\left[H^{\sharp}\left(\xi_{1}\right), H^{\sharp}\left(\xi_{2}\right)\right]_{A}\right\rangle, \quad \forall \xi_{1}, \xi_{2}, \xi_{3} \in \Gamma\left(A^{*}\right)(39)
\end{aligned}
$$

Suppose that $H^{\sharp}: A^{*} \longrightarrow A$ is nondegenerate. Then $\left(H^{\sharp}\right)^{-1}: A \longrightarrow A^{*}$ is also a symmetric bundle map, which gives rise to an element, denoted by $H^{-1}$, in $\operatorname{Sym}^{2}\left(A^{*}\right)$.

Proposition 4.6. Let $\left(A, \cdot A, a_{A}\right)$ be a left-symmetric algebroid and $H \in \operatorname{Sym}^{2}(A)$. If $H$ is nondegenerate, then the following two statements are equivalent:

(1) $\llbracket H, H \rrbracket=0$;

(2) $\delta\left(H^{-1}\right)=0$.

Proof. By direct calculation, we have the following formula

$$
\delta\left(H^{-1}\right)\left(H^{\sharp}\left(\xi_{1}\right), H^{\sharp}\left(\xi_{2}\right), H^{\sharp}\left(\xi_{3}\right)\right)=\llbracket H, H \rrbracket\left(\xi_{1}, \xi_{2}, \xi_{3}\right), \quad \forall \xi_{1}, \xi_{2}, \xi_{3} \in \Gamma\left(A^{*}\right) .
$$

Thus, the conclusion follows immediately.

Let $\left(A, \cdot_{A}, a_{A}\right)$ be a left-symmetric algebroid, and $H \in \operatorname{Sym}^{2}(A)$. Define

$$
\xi \cdot{ }_{H} \eta=\mathcal{L}_{H^{\sharp}(\xi)} \eta-R_{H^{\sharp}(\eta)} \xi-\mathrm{d}(H(\xi, \eta)), \quad \forall \xi, \eta \in \Gamma\left(A^{*}\right) .
$$

Proposition 4.7. With the above notations, for all $\xi, \eta \in \Gamma\left(A^{*}\right)$, we have

$$
H^{\sharp}(\xi \cdot H \eta)-H^{\sharp}(\xi) \cdot{ }_{A} H^{\sharp}(\eta)=\llbracket H, H \rrbracket(\xi, \cdot, \eta) .
$$

Proof. First, for all $\xi, \eta, \zeta \in \Gamma\left(A^{*}\right)$, we have

$$
\begin{aligned}
\left\langle H^{\sharp}(\xi \cdot H \eta), \zeta\right\rangle= & \left\langle\xi \cdot H \eta, H^{\sharp}(\zeta)\right\rangle=\left\langle\mathcal{L}_{H^{\sharp}(\xi)} \eta-R_{H^{\sharp}(\eta)} \xi-\mathrm{d}(H(\xi, \eta)), H^{\sharp}(\zeta)\right\rangle \\
= & a_{A}\left(H^{\sharp}(\xi)\right)\left\langle H^{\sharp}(\zeta), \eta\right\rangle-a_{A}\left(H^{\sharp}(\zeta)\right)\left\langle H^{\sharp}(\xi), \eta\right\rangle+\left\langle\xi, H^{\sharp}(\zeta) \cdot{ }_{A} H^{\sharp}(\eta)\right\rangle \\
& -\left\langle\eta,\left[H^{\sharp}(\xi), H^{\sharp}(\zeta)\right]\right\rangle .
\end{aligned}
$$

Thus, by (39), we have

$$
\left\langle H^{\sharp}\left(\xi \cdot{ }_{H} \eta\right), \zeta\right\rangle-\left\langle H^{\sharp}(\xi) \cdot{ }_{A} H^{\sharp}(\eta), \zeta\right\rangle=\llbracket H, H \rrbracket(\xi, \zeta, \eta),
$$

which finishes the proof.

By direct calculation, we have

Corollary 4.8. For all $\xi, \eta \in \Gamma\left(A^{*}\right)$, we have

$$
H^{\sharp}\left([\xi, \eta]_{H}\right)-\left[H^{\sharp}(\xi), H^{\sharp}(\eta)\right]_{A}=\llbracket H, H \rrbracket(\xi, \eta, \cdot),
$$

where $[\cdot, \cdot]_{H}$ is the commutator bracket of $\cdot_{H}$.

Theorem 4.9. With the above notations, if $\llbracket H, H \rrbracket=0$, then $\left(A^{*}, \cdot_{H}, a_{A^{*}}=a_{A} \circ H^{\sharp}\right)$ is a leftsymmetric algebroid, and $H^{\sharp}$ is a left-symmetric algebroid homomorphism from $\left(A^{*}, \cdot_{H}, a_{A^{*}}\right)$ to $\left(A, \cdot A, a_{A}\right)$. Furthermore, $\left(A, A^{*}\right)$ is a left-symmetric bialgebroid. 
Proof. By direct calculation, we can show that $\left(\Gamma\left(A^{*}\right), \cdot H\right)$ is a left-symmetric algebra. Moreover, we have

$$
\begin{aligned}
& \xi \cdot H \quad f \eta=\left(f \mathcal{L}_{H^{\sharp} \xi} \eta+a_{A} \circ H^{\sharp}(\xi)(f) \eta\right)-\left(f R_{H^{\sharp} \eta} \xi-\left\langle H^{\sharp}(\xi), \eta\right\rangle \mathrm{d} f\right)-(f \mathrm{~d} H(\xi, \eta)+H(\xi, \eta) \mathrm{d} f) \\
& =f(\xi \cdot H \eta)+a_{A} \circ H^{\sharp}(\xi)(f) \eta ; \\
& (f \xi) \cdot H \quad=\left(f \mathcal{L}_{H^{\sharp} \xi} \eta+\left\langle H^{\sharp}(\xi), \eta\right\rangle \mathrm{d} f\right)-f R_{H^{\sharp} \eta} \xi-(f \mathrm{~d} H(\xi, \eta)+H(\xi, \eta) \mathrm{d} f) \\
& =f(\xi \cdot H \eta) \text {. }
\end{aligned}
$$

Thus, $\left(A^{*}, \cdot_{H}, a_{A} \circ H\right)$ is a left-symmetric algebroid. By (41), $H^{\sharp}$ is a left-symmetric algebroid homomorphism.

To obtain that $\left(A, A^{*}\right)$ is a left-symmetric bialgebroid, we need to prove that (32) and (33) hold. By tedious calculation, (32) can be obtained directly and (33) is equivalent to the following equation:

$$
a_{A}\left[H^{\sharp}(\xi), x\right]=a_{A}\left(L_{\xi}^{*} x-H^{\sharp}\left(L_{x}^{*} \xi\right)\right) .
$$

By direct calculation, we have

$$
\begin{aligned}
\left\langle L_{\xi}^{*} x-H^{\sharp}\left(L_{x}^{*} \xi\right), \eta\right\rangle= & a_{A^{*}}(\xi)\langle x, \eta\rangle-\langle x, \xi \cdot H \eta\rangle-a_{A}(x)\left\langle\xi, H^{\sharp}(\eta)\right\rangle+\left\langle\xi, x \cdot{ }_{A} H^{\sharp}(\eta)\right\rangle \\
= & a_{A^{*}}(\xi)\langle x, \eta\rangle-a_{A}\left(H^{\sharp}(\xi)\right)\langle x, \eta\rangle+a_{A}(x)\left\langle H^{\sharp}(\xi), \eta\right\rangle+\left\langle\eta,\left[H^{\sharp}(\xi), x\right]\right\rangle \\
& -\left\langle\xi, x \cdot{ }_{A} H^{\sharp}(\eta)\right\rangle-a_{A}(x)\left\langle\xi, H^{\sharp}(\eta)\right\rangle+\left\langle\xi, x \cdot{ }_{A} H^{\sharp}(\eta)\right\rangle \\
= & \left\langle\left[H^{\sharp}(\xi), x\right], \eta\right\rangle,
\end{aligned}
$$

which implies that (43) holds.

Remark 4.10. When $M$ is a point, $\llbracket H, H \rrbracket=0$ is exactly the $S$-equation introduced in [1]. Thus, the equation $\llbracket H, H \rrbracket=0$ can be viewed as a geometric generalization of the S-equation.

At the end of this section, we give some applications in Hessian geometry and construct a left-symmetric bialgebroid from a pseudo-Hessian manifold.

Recall that a pseudo-Hessian metric $g$ is a pseudo-Riemannian metric $g$ on a flat manifold $(M, \nabla)$ such that $g$ can be locally expressed by $g_{i j}=\frac{\partial^{2} \varphi}{\partial x^{2} \partial x^{j}}$, where $\varphi \in C^{\infty}(M)$ and $\left\{x^{1}, \cdots, x^{n}\right\}$ is an affine coordinate system with respect to $\nabla$, i.e.

$$
\nabla_{\frac{\partial}{\partial x^{i}}} \frac{\partial}{\partial x^{i}}=0, i=1, \ldots, n .
$$

Then the pair $(\nabla, g)$ is called a pseudo-Hessian structure on $M$. A manifold $M$ with a pseudoHessian structure $(\nabla, g)$ is called a pseudo-Hessian manifold. See [26] for more details about pseudo-Hessian manifolds.

Proposition 4.11. 26] Let $(M, \nabla)$ be a flat manifold and $g$ a pseudo-Riemannian metric on $M$. Then the following conditions are equivalent:

(1) $\mathfrak{g}$ is a pseudo-Hessian metric;

(2) for all $x, y, z \in \Gamma(T M)$, there holds $\nabla_{x} g(y, z)=\nabla_{y} g(x, z)$, where $\nabla_{x} g(y, z)$ is given by

$$
\nabla_{x} g(y, z)=x g(y, z)-g\left(\nabla_{x} y, z\right)-g\left(y, \nabla_{x} z\right) .
$$


Proposition 4.12. Let $(M, \nabla)$ be a flat manifold and $g$ a pseudo-Riemannian metric on $M$. Then $(M, \nabla, g)$ is a pseudo-Hessian manifold if and only if $\delta g(x, y, z)=0$, where $\delta$ is the coboundary operator given by (7) associated to the left-symmetric algebroid $T_{\nabla} M$ given in Example 2.4.

Proof. By Proposition 4.11 and the following formula

$$
\delta g(x, y, z)=\nabla_{x} g(y, z)-\nabla_{y} g(x, z),
$$

we can obtain the conclusion immediately.

By Proposition 4.12, Proposition 4.6 and Theorem 4.9, we can construct a left-symmetric bialgebroid from a pseudo-Hessian manifold.

Proposition 4.13. Let $(M, \nabla, g)$ be a pseudo-Hessian manifold. Define $H \in \operatorname{Sym}^{2}(T M)$ by

$$
\left(H^{\sharp}\right)^{-1}(x)(y)=g(x, y) .
$$

Then $\left(T^{*} M, \cdot_{H}, H^{\sharp}\right)$ is a left-symmetric algebroid, which we denote by $T_{H}^{*} M$, where $\cdot_{H}$ is given by (40). Furthermore, $H^{\sharp}$ is a left-symmetric algebroid homomorphism from $T_{H}^{*} M$ to $T_{\nabla} M$ and $\left(T_{\nabla} M, T_{H}^{*} M\right)$ is a left-symmetric bialgebroid.

Remark 4.14. The above result is parallel to that $\left(T M, T_{\pi}^{*} M\right)$ is a Lie bialgebroid for any Poisson manifold $(M, \pi)$. See [9] for more details.

\section{Equivalence between Manin triples for left-symmetric al- gebroids and left-symmetric bialgebroids}

In this section, we introduce the notion of a Manin triple for left-symmetric algebroids as a geometric generalization of a Manin triple for left-symmetric algebras. We would like to point out that unlike the latter, the double structure of the former is a pre-symplectic algebroid rather than a left-symmetric algebroid. We show that Manin triples for left-symmetric algebroids are equivalent to left-symmetric bialgebroids. At the end of this section, we establish a relation between Maurer-Cartan type equations and Dirac structures of the pre-symplectic algebroid which is the corresponding double structure for a left-symmetric bialgebroid.

Definition 5.1. A Manin triple for left-symmetric algebroids is a triple $\left(E ; L_{1}, L_{2}\right)$, where $E$ is a pre-symplectic algebroid, $L_{1}$ and $L_{2}$ are transversal Dirac structures.

Example 5.2. Let $\left(A, \cdot A, a_{A}\right)$ be a left-symmetric algebroid and $\left(A \oplus A^{*}, \star, \rho,(\cdot, \cdot)_{-}\right)$the corresponding pre-symplectic algebroid given in Example 2.10. Then $\left(A \oplus A^{*} ; A, A^{*}\right)$ is a Manin triple for left-symmetric algebroids.

More generally, we have

Theorem 5.3. There is a one-to-one correspondence between Manin triples for left-symmetric algebroids and left-symmetric bialgebroids.

Proof. Follows form the following Proposition 5.4 and Proposition 5.5.

Suppose that both $\left(A, \cdot_{A}, a_{A}\right)$ and $\left(A^{*}, A^{*}, a_{A^{*}}\right)$ are left-symmetric algebroids. Let $E=A \oplus A^{*}$. We introduce a multiplication $\star: \Gamma(E) \times \Gamma(E) \longrightarrow \Gamma(E)$ by

$$
e_{1} \star e_{2}=\left(x_{1} \cdot{ }_{A} x_{2}+\mathcal{L}_{\xi_{1}} x_{2}-R_{\xi_{2}} x_{1}-\frac{1}{2} \mathrm{~d}_{*}\left(e_{1}, e_{2}\right)_{+}\right)+\left(\xi_{1} \cdot A^{*} \xi_{2}+\mathcal{L}_{x_{1}} \xi_{2}-R_{x_{2}} \xi_{1}-\frac{1}{2} \mathrm{~d}\left(e_{1}, e_{2}\right)_{+}\right),
$$


where $e_{1}=x_{1}+\xi_{1}, e_{2}=x_{2}+\xi_{2}, \mathcal{L}$ is given by (10), $R$ is given by (23) and $(\cdot, \cdot)_{+}$is given by (14). Let $\rho: E \rightarrow T M$ be the bundle map defined by $\rho=a_{A}+a_{A^{*}}$. That is,

$$
\rho(x+\xi)=a_{A}(x)+a_{A^{*}}(\xi), \forall x \in \Gamma(A), \xi \in \Gamma\left(A^{*}\right) .
$$

It is obvious that in this case the operator $D$ (see (9)) is given by

$$
D=\mathrm{d}-\mathrm{d}_{*},
$$

where d : $C^{\infty}(M) \rightarrow \Gamma\left(A^{*}\right)$ and $\mathrm{d}_{*}: C^{\infty}(M) \rightarrow \Gamma(A)$ are the usual differential operators associated to the sub-adjacent Lie algebroids $A^{c}$ and $A^{* c}$ respectively.

Proposition 5.4. With the above notations, let $\left(A, A^{*}\right)$ be a left-symmetric bialgebroid. Then $\left(A \oplus A^{*}, \star, \rho,(\cdot, \cdot)_{-}\right)$is a pre-symplectic algebroid, where the multiplication $\star$ is given by (44), $\rho=$ $a_{A}+a_{A^{*}}$, and $(\cdot, \cdot)_{-}$is given by (5).

Conversely, we have

Proposition 5.5. Let $\left(E, \star, \rho,(\cdot, \cdot)_{-}\right)$be a pre-symplectic algebroid. Suppose that $L_{1}$ and $L_{2}$ are Dirac subbundles transversal to each other. Then $\left(L_{1}, L_{2}\right)$ is a left-symmetric bialgebroid, where $L_{2}$ is considered as the dual bundle of $L_{1}$ under the nondegenerate bilinear form $(\cdot, \cdot)_{-}$.

We prove some lemmas first.

Lemma 5.6. Let $\left(A, \cdot_{A}, a_{A}\right)$ and $\left(A^{*}, \cdot A^{*}, a_{A^{*}}\right)$ be two left-symmetric algebroids. For all $e_{i}=$ $x_{i}+\xi_{i} \in \Gamma(E), i=1,2,3,4$, we have

$$
\left(\left(x_{1}, x_{2}, \xi_{3}\right)-\left(x_{2}, x_{1}, \xi_{3}\right), x_{4}+\xi_{4}\right)_{-}=\frac{1}{6}\left(D T\left(x_{1}, x_{2}, \xi_{3}\right), x_{4}+\xi_{4}\right)_{-}-I_{1}-I_{2}+I_{3},
$$

where $I_{1}, I_{2}, I_{3}$ is defined by

$$
\begin{aligned}
& I_{1}=\left\langle\delta_{*}\left[x_{1}, x_{2}\right]_{A}-\mathfrak{L}_{x_{1}} \delta_{*} x_{2}+\mathfrak{L}_{x_{2}} \delta_{*} x_{1}, \xi_{4} \otimes \xi_{3}\right\rangle ; \\
& I_{2}=\left(\left[a_{A}\left(x_{1}\right), a_{A^{*}}\left(\xi_{4}\right)\right]-a_{A^{*}}\left(L_{x_{1}}^{*} \xi_{4}\right)+a_{A}\left(L_{\xi_{1}}^{*} x_{4}\right)\right)\left\langle x_{2}, \xi_{3}\right\rangle ; \\
& I_{3}=\left(\left[a_{A}\left(x_{2}\right), a_{A^{*}}\left(\xi_{4}\right)\right]-a_{A^{*}}\left(L_{x_{2}}^{*} \xi_{4}\right)+a_{A}\left(L_{\xi_{4}}^{*} x_{2}\right)\right)\left\langle x_{1}, \xi_{3}\right\rangle .
\end{aligned}
$$

Proof. First, we have

$$
\begin{aligned}
& \left(x_{1}, x_{2}, \xi_{3}\right)-\left(x_{2}, x_{1}, \xi_{3}\right) \\
= & \mathcal{L}_{x_{1}} \mathcal{L}_{x_{2}} \xi_{3}-\mathcal{L}_{x_{2}} L_{x_{1}}^{*} \xi_{3}-\mathcal{L}_{\left[x_{1}, x_{2}\right]_{A} \xi_{3}+\left(-x_{1} \cdot{ }_{A} R_{\xi_{3}} x_{2}+x_{2} \cdot{ }_{A} R_{\xi_{3}} x_{1}\right.} \\
& \left.-R_{\mathcal{L}_{x_{2}} \xi_{3}} x_{1}+R_{L_{x_{1}}^{*} \xi_{3}} x_{2}+R_{\xi_{3}}\left[x_{1}, x_{2}\right]_{A}\right)-\frac{1}{2} x_{1} \cdot{ }_{A} \mathrm{~d}_{*}\left\langle x_{2}, \xi_{3}\right\rangle+\frac{1}{2} x_{2} \cdot{ }_{A} \mathrm{~d}_{*}\left\langle x_{1}, \xi_{3}\right\rangle \\
& -\frac{1}{2} \mathrm{~d}_{*}\left\langle x_{1}, \mathcal{L}_{x_{2}} \xi_{3}\right\rangle+\frac{1}{2} \mathrm{~d}_{*}\left\langle x_{2}, \mathcal{L}_{x_{1}} \xi_{3}\right\rangle+\frac{1}{2} R_{\mathrm{d}\left\langle x_{2}, \xi_{3}\right\rangle} x_{1}-\frac{1}{2} R_{\mathrm{d}\left\langle x_{1}, \xi_{3}\right\rangle} x_{2}+\frac{1}{2} \mathrm{~d}_{*}\left\langle\left[x_{1}, x_{2}\right], \xi_{3}\right\rangle \\
& +\frac{1}{4} \mathrm{~d}_{*}\left\langle x_{1}, \mathrm{~d}\left\langle x_{2}, \xi_{3}\right\rangle\right\rangle-\frac{1}{4} \mathrm{~d}_{*}\left\langle x_{2}, \mathrm{~d}\left\langle x_{1}, \xi_{3}\right\rangle\right\rangle-\frac{1}{2} \mathcal{L}_{x_{1}} \mathrm{~d}\left\langle x_{2}, \xi_{3}\right\rangle+\frac{1}{2} \mathcal{L}_{x_{2}} \mathrm{~d}\left\langle x_{1}, \xi_{3}\right\rangle \\
& -\frac{1}{2} \mathrm{~d}\left\langle x_{1}, \mathcal{L}_{x_{2}} \xi_{3}\right\rangle+\frac{1}{2} \mathrm{~d}\left\langle x_{2}, \mathcal{L}_{x_{1}} \xi_{3}\right\rangle-\frac{1}{4} \mathrm{~d}\left\langle x_{2}, \mathrm{~d}\left\langle x_{1}, \xi_{3}\right\rangle\right\rangle+\frac{1}{4} \mathrm{~d}\left\langle x_{1}, \mathrm{~d}\left\langle x_{2}, \xi_{3}\right\rangle\right\rangle+\frac{1}{2} \mathrm{~d}\left\langle\left[x_{1}, x_{2}\right]_{A}, \xi_{3}\right\rangle .
\end{aligned}
$$

By direct calculation, we have

$$
\begin{aligned}
& \left\langle\delta_{*}\left[x_{1}, x_{2}\right]_{A}-\mathfrak{L}_{x_{1}} \delta_{*} x_{2}+\mathfrak{L}_{x_{2}} \delta_{*} x_{1}, \xi_{4} \otimes \xi_{3}\right\rangle \\
= & \left\langle-x_{1} \cdot A R_{\xi_{3}} x_{2}+x_{2} \cdot{ }_{A} R_{\xi_{3}} x_{1}-R_{\mathcal{L}_{x_{2}} \xi_{3}} x_{1}+R_{\mathcal{L}_{x_{1}} \xi_{3}} x_{2}+R_{\xi_{3}}\left[x_{1}, x_{2}\right]_{A}, \xi_{4}\right\rangle \\
& +a_{A^{*}}\left(\xi_{4}\right)\left\langle\left[x_{1}, x_{2}\right]_{A}, \xi_{3}\right\rangle+\left(\left[a_{A}\left(x_{1}\right), a_{A^{*}}\left(\xi_{4}\right)\right]+a_{A^{*}}\left(L_{x_{1}}^{*} \xi_{4}\right)\right)\left\langle x_{2}, \xi_{3}\right\rangle \\
& -\left(\left[a_{A}\left(x_{2}\right), a_{A^{*}}\left(\xi_{4}\right)\right]+a_{A^{*}}\left(L_{x_{2}}^{*} \xi_{4}\right)\right)\left\langle x_{1}, \xi_{3}\right\rangle .
\end{aligned}
$$


Therefore, we obtain

$$
\begin{aligned}
& \left(\left(x_{1}, x_{2}, \xi_{3}\right)-\left(x_{2}, x_{1}, \xi_{3}\right), x_{4}+\xi_{4}\right)_{-} \\
= & -\left\langle\delta_{*}\left[x_{1}, x_{2}\right]_{A}-\mathfrak{L}_{x_{1}} \delta_{*} x_{2}+\mathfrak{L}_{x_{2}} \delta_{*} x_{1}, \xi_{4} \otimes \xi_{3}\right\rangle \\
& -\left(\left[a_{A}\left(x_{1}\right), a_{A^{*}}\left(\xi_{4}\right)\right]-a_{A^{*}}\left(L_{x_{1}}^{*} \xi_{4}\right)+a_{A}\left(L_{\xi_{1}}^{*} x_{4}\right)\right)\left\langle x_{2}, \xi_{3}\right\rangle \\
& +\left(\left[a_{A}\left(x_{2}\right), a_{A^{*}}\left(\xi_{4}\right)\right]-a_{A^{*}}\left(L_{x_{2}}^{*} \xi_{4}\right)+a_{A}\left(L_{\xi_{4}}^{*} x_{2}\right)\right)\left\langle x_{1}, \xi_{3}\right\rangle \\
& +\frac{1}{4}\left(\left(\mathrm{~d}-\mathrm{d}_{*}\right)\left(a_{A}\left(x_{1}\right)\left\langle x_{2}, \xi_{3}\right\rangle-a_{A}\left(x_{2}\right)\left\langle x_{1}, \xi_{3}\right\rangle-2\left\langle\left[x_{1}, x_{2}\right]_{A}, \xi_{3}\right\rangle\right), x_{4}+\xi_{4}\right)_{-} .
\end{aligned}
$$

It is easy to see that

$$
\begin{aligned}
& \left(\frac{1}{6}\left\langle D T\left(x_{1}, x_{2}, \xi_{3}\right), x_{4}+\xi_{4}\right)_{-}\right. \\
= & \frac{1}{4}\left(\left(\mathrm{~d}-\mathrm{d}_{*}\right)\left(a_{A}\left(x_{1}\right)\left\langle x_{2}, \xi_{3}\right\rangle-a_{A}\left(x_{2}\right)\left\langle x_{1}, \xi_{3}\right\rangle-2\left\langle\left[x_{1}, x_{2}\right]_{A}, \xi_{3}\right\rangle\right), x_{4}+\xi_{4}\right)_{-} .
\end{aligned}
$$

The proof is finished.

Lemma 5.7. With the above notations, we have

$$
\left(\left(x_{1}, \xi_{2}, x_{3}\right)-\left(\xi_{2}, x_{1}, x_{3}\right), x_{4}+\xi_{4}\right)_{-}=\frac{1}{6}\left(D T\left(x_{1}, \xi_{2}, x_{3}\right), x_{4}+\xi_{4}\right)+J_{1}-J_{2}-J_{3},
$$

where $J_{1}, J_{2}, J_{3}$ is defined by

$$
\begin{aligned}
J_{1} & =\left\langle\delta_{*}\left[\xi_{2}, \xi_{4}\right]_{A}-\mathfrak{L}_{\xi_{2}} \delta_{*} \xi_{4}+\mathfrak{L}_{\xi_{4}} \delta_{*} \xi_{2}, x_{1} \otimes x_{3}\right\rangle ; \\
J_{2} & =\left(\left[a_{A^{*}}\left(\xi_{2}\right), a_{A}\left(x_{1}\right)\right]-a_{A}\left(L_{\xi_{2}}^{*} x_{1}\right)+a_{A^{*}}\left(L_{x_{1}}^{*} \xi_{2}\right)\right)\left\langle\xi_{4}, x_{3}\right\rangle ; \\
J_{3} & =\frac{1}{2}\left(\left[a_{A^{*}}\left(\xi_{4}\right), a_{A}\left(x_{1}\right)\right]-a_{A}\left(L_{\xi_{4}}^{*} x_{1}\right)+a_{A^{*}}\left(L_{x_{1}}^{*} \xi_{4}\right)\right)\left\langle\xi_{2}, x_{3}\right\rangle .
\end{aligned}
$$

Proof. First we have

$$
\begin{aligned}
& \left(x_{1}, \xi_{2}, x_{3}\right)-\left(\xi_{2}, x_{1}, x_{3}\right) \\
= & \left(-\mathcal{L}_{x_{1}} R_{x_{3}} \xi_{2}+R_{x_{3}} \mathcal{L}_{x_{1}} \xi_{2}+R_{x_{1} \cdot A} x_{3} \xi_{3}+R_{x_{3}} R_{x_{1}} \xi_{2}\right)+x_{1} \cdot{ }_{A} \mathcal{L}_{\xi_{2}} x_{3}+R_{R_{x_{3}} \xi_{2} x_{1}} \\
& -\mathcal{L}_{\mathcal{L}_{x} \xi_{2}} x_{3}+\left(R_{\xi_{2}} x_{1}\right) \cdot{ }_{A} x_{3}-\mathcal{L}_{\xi_{2}}\left(x_{1} \cdot{ }_{A} x_{3}\right)+\mathcal{L}_{\xi_{2}} x_{1} \cdot{ }_{A} x_{3}-\mathcal{L}_{R_{x_{1}} \xi_{2}} x_{3} \\
& -\frac{1}{2} x_{1} \cdot{ }_{A} \mathrm{~d}_{*}\left\langle\xi_{2}, x_{3}\right\rangle+\frac{1}{2} R_{\mathrm{d}\left\langle\xi_{2}, x_{3}\right\rangle} x_{1}+\frac{1}{2} \mathrm{~d}_{*}\left\langle\mathcal{L}_{x_{1}} \xi_{2}, x_{3}\right\rangle+\frac{1}{2} \mathrm{~d}_{*}\left\langle R_{x_{1}} \xi_{2}, x_{3}\right\rangle \\
& -\frac{1}{2} \mathcal{L}_{\mathrm{d}\left\langle\xi_{2}, x_{1}\right\rangle} x_{3}+\frac{1}{4} \mathrm{~d}_{*}\left\langle x_{1}, \mathrm{~d}\left\langle\xi_{2}, x_{3}\right\rangle\right\rangle-\frac{1}{2} \mathcal{L}_{x_{1}} \mathrm{~d}\left\langle\xi_{2}, x_{3}\right\rangle \\
& +\frac{1}{2} \mathrm{~d}\left\langle\mathcal{L}_{x_{1}} \xi_{2}, x_{3}\right\rangle+\frac{1}{2} \mathrm{~d}\left\langle R_{x_{1}} \xi_{2}, x_{3}\right\rangle+\frac{1}{4} \mathrm{~d}\left\langle x_{1}, \mathrm{~d}\left\langle\xi_{2}, x_{3}\right\rangle\right\rangle .
\end{aligned}
$$

Similarly, by direct calculation, we have

$$
\begin{aligned}
& \left\langle\delta\left[\xi_{2}, \xi_{4}\right]_{A}-\mathfrak{L}_{\xi_{1}} \delta \xi_{4}+\mathfrak{L}_{\xi_{4}} \delta \xi_{2}, x_{1} \otimes x_{3}\right\rangle \\
= & -\left\langle x_{1} \cdot{ }_{A} \mathcal{L}_{\xi_{2}} x_{3}+R_{R_{x_{3}} \xi_{2}} x_{1}-\mathcal{L}_{\mathcal{L}_{x} \xi_{2}} x_{3}+\left(R_{\xi_{2}} x_{1}\right) \cdot{ }_{A} x_{3}-\mathcal{L}_{\xi_{2}}\left(x_{1} \cdot{ }_{A} x_{3}\right), \xi_{4}\right\rangle \\
& +\left(\left[a_{A^{*}}\left(\xi_{2}\right), a_{A}\left(x_{1}\right)\right]-a_{A}\left(L_{\xi_{2}}^{*} x_{1}\right)+a_{A^{*}}\left(L_{x_{1}}^{*} \xi_{2}\right)\right)\left\langle\xi_{4}, x_{3}\right\rangle+a_{A^{*}}\left(\xi_{4}\right)\left\langle\xi_{2}, x_{1} \cdot{ }_{A} x_{3}\right\rangle .
\end{aligned}
$$


Therefore, we obtain

$$
\begin{aligned}
& \left(\left(x_{1}, \xi_{2}, x_{3}\right)-\left(\xi_{2}, x_{1}, x_{3}\right), x_{4}+\xi_{4}\right)_{-} \\
= & +\left\langle\delta\left[\xi_{2}, \xi_{4}\right]_{A}-\mathfrak{L}_{\xi_{1}} \delta \xi_{4}+\mathfrak{L}_{\xi_{4}} \delta \xi_{2}, x_{1} \otimes x_{3}\right\rangle \\
& -\left(\left[a_{A^{*}}\left(\xi_{2}\right), a_{A}\left(x_{1}\right)\right]-a_{A}\left(L_{\xi_{2}}^{*} x_{1}\right)+a_{A^{*}}\left(L_{x_{1}}^{*} \xi_{2}\right)\right)\left\langle\xi_{4}, x_{3}\right\rangle \\
& -\frac{1}{2}\left(\left[a_{A^{*}}\left(\xi_{4}\right), a_{A}\left(x_{1}\right)\right]-a_{A}\left(L_{\xi_{4}}^{*} x_{1}\right)+a_{A^{*}}\left(L_{x_{1}}^{*} \xi_{4}\right)\right)\left\langle\xi_{2}, x_{3}\right\rangle \\
& +\frac{1}{4}\left(\left(\mathrm{~d}-\mathrm{d}_{*}\right)\left(a_{A}\left(x_{1}\right)\left\langle\xi_{2}, x_{3}\right\rangle-2\left\langle x_{1} \cdot{ }_{A} x_{3}, \xi_{2}\right\rangle, x_{4}+\xi_{4}\right)_{-} \cdot\right.
\end{aligned}
$$

It is easy to see that

$$
\frac{1}{6}\left(D T\left(x_{1}, \xi_{2}, x_{3}\right), x_{4}+\xi_{4}\right)_{-}=\frac{1}{4}\left(\left(\mathrm{~d}-\mathrm{d}_{*}\right)\left(a_{A}\left(x_{1}\right)\left\langle\xi_{2}, x_{3}\right\rangle-2\left\langle x_{1} \cdot A x_{3}, \xi_{2}\right\rangle\right), x_{4}+\xi_{4}\right)_{-} .
$$

The proof is finished.

Lemma 5.8. With the above notations, we have

$$
\left.\left(\xi_{1}, x_{2}, x_{3}\right)-\left(x_{2}, \xi_{1}, x_{3}\right), x_{4}+\xi_{4}\right)_{-}=\frac{1}{6}\left(D T\left(\xi_{1}, x_{2}, x_{3}\right)_{-}, x_{4}+\xi_{4}\right)-J_{1}+J_{2}+J_{3},
$$

where $J_{1}, J_{2}, J_{3}$ is defined by

$$
\begin{aligned}
J_{1} & =\left\langle\delta_{*}\left[\xi_{1}, \xi_{4}\right]_{A}-\mathfrak{L}_{\xi_{1}} \delta_{*} \xi_{4}+\mathfrak{L}_{\xi_{4}} \delta_{*} \xi_{1}, x_{1} \otimes x_{3}\right\rangle \\
J_{2} & =\left(\left[a_{A^{*}}\left(\xi_{1}\right), a_{A}\left(x_{2}\right)\right]-a_{A}\left(L_{\xi_{1}}^{*} x_{2}\right)+a_{A^{*}}\left(L_{x_{2}}^{*} \xi_{1}\right)\right)\left\langle\xi_{4}, x_{3}\right\rangle ; \\
J_{3} & =\frac{1}{2}\left(\left[a_{A^{*}}\left(\xi_{4}\right), a_{A}\left(x_{2}\right)\right]-a_{A}\left(L_{\xi_{4}}^{*} x_{2}\right)+a_{A^{*}}\left(L_{x_{2}}^{*} \xi_{4}\right)\right)\left\langle\xi_{1}, x_{3}\right\rangle .
\end{aligned}
$$

Proof. By Lemma 5.7 and $T\left(e_{1}, e_{2}, e_{3}\right)=-T\left(e_{2}, e_{1}, e_{3}\right)$. The lemma follows immediately.

Proof of Proposition 5.4 To prove Proposition 5.4, it is sufficient to verify that conditions (i) and (ii) in Definition 2.7 hold. First, condition (i) in Definition 2.7 follows directly from Lemma 5.6 -5.8 and properties of left-symmetric bialgebroids. Below, we show that condition (ii) in Definition 2.7 holds. On one hand, we have

$$
\rho\left(e_{1}\right)\left(e_{2}, e_{3}\right)_{-}=\left(a_{A}\left(x_{1}\right)+a_{A^{*}}\left(\xi_{1}\right)\right)\left(\left\langle\xi_{2}, x_{3}\right\rangle-\left\langle x_{2}, \xi_{3}\right\rangle\right), \quad \forall e_{i}=x_{i}+\xi_{i} \in \Gamma(E), i=1,2,3 .
$$

On the other hand, we have

$$
\begin{aligned}
\left(e_{1} \star e_{2}-\frac{1}{2} D\left(e_{1}, e_{2}\right)_{-}, e_{3}\right)_{-}= & \left\langle\xi_{1} \cdot A^{*} \xi_{2}, x_{3}\right\rangle-\left\langle\xi_{2},\left[x_{1}, x_{3}\right]_{A}\right\rangle+\left\langle\xi_{1}, x_{3} \cdot A x_{2}\right\rangle-\left\langle\xi_{3}, x_{1} \cdot A x_{2}\right\rangle \\
& +\left\langle x_{2},\left[\xi_{1}, \xi_{3}\right]_{A}\right\rangle-\left\langle\xi_{3} \cdot A^{*} \xi_{2}, x_{1}\right\rangle+a_{A}\left(x_{1}\right)\left\langle\xi_{2}, x_{3}\right\rangle \\
& -a_{A^{*}}\left(\xi_{1}\right)\left\langle x_{2}, \xi_{3}\right\rangle-a_{A}\left(x_{3}\right)\left\langle\xi_{1}, x_{2}\right\rangle+a_{A^{*}}\left(\xi_{3}\right)\left\langle\xi_{2}, x_{1}\right\rangle
\end{aligned}
$$

and

$$
\begin{aligned}
\left(e_{2},\left[e_{1}, e_{3}\right]_{E}\right)_{-}= & -\left(\left\langle\xi_{1} \cdot A^{*} \xi_{2}, x_{3}\right\rangle-\left\langle\xi_{2},\left[x_{1}, x_{3}\right]_{A}\right\rangle+\left\langle\xi_{1}, x_{3} \cdot x_{A} x_{2}\right\rangle\right. \\
& \left.-\left\langle\xi_{3}, x_{1} \cdot A x_{2}\right\rangle+\left\langle x_{2},\left[\xi_{1}, \xi_{3}\right]_{A}\right\rangle-\left\langle\xi_{3} \cdot A^{*} \xi_{2}, x_{1}\right\rangle\right) \\
& +a_{A^{*}}\left(\xi_{1}\right)\left\langle\xi_{2}, x_{3}\right\rangle-a_{A^{*}}\left(\xi_{3}\right)\left\langle\xi_{2}, x_{1}\right\rangle-a_{A}\left(x_{1}\right)\left\langle x_{2}, \xi_{3}\right\rangle+a_{A}\left(x_{3}\right)\left\langle\xi_{1}, x_{2}\right\rangle,
\end{aligned}
$$


which implies that condition (ii) in Definition 2.7 holds.

Proof of Proposition 5.5. Since the pairing $(\cdot, \cdot)_{-}$is nondegenerate, $L_{2}$ is isomorphic to $L_{1}^{*}$,

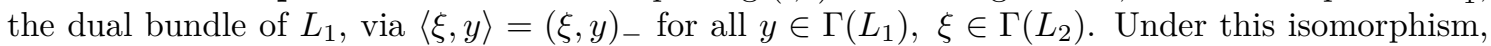
the skew-symmetric bilinear form $(\cdot, \cdot)_{-}$on $E$ is given by

$$
(\xi+x, y+\eta)_{-}=\langle\xi, y\rangle-\langle y, \eta\rangle .
$$

By Proposition 2.12, both $L_{1}$ and $L_{2}$ are left-symmetric algebroids, their anchors are given by $a=\left.\rho\right|_{L_{1}}$ and $a_{*}=\left.\rho\right|_{L_{2}}$ respectively. We shall use $\delta, \mathrm{d}$ and $\delta_{*}, \mathrm{~d}_{*}$ to denote their differential of left-symmetric algebroids and corresponding sub-adjacent Lie algebroids respectively.

By condition (ii) in Definition 2.7, we deduce that the bracket between $x \in \Gamma\left(L_{1}\right)$ and $\xi \in \Gamma\left(L_{2}\right)$ is given by

$$
\begin{aligned}
& x \star \xi=\mathcal{L}_{x} \xi+\frac{1}{2} \mathrm{~d}\langle x, \xi\rangle-R_{\xi} x+\frac{1}{2} \mathrm{~d}_{*}\langle x, \xi\rangle ; \\
& \xi \star x=\mathcal{L}_{\xi} x-\frac{1}{2} \mathrm{~d}_{*}\langle x, \xi\rangle-R_{x} \xi-\frac{1}{2} \mathrm{~d}\langle x, \xi\rangle .
\end{aligned}
$$

Thus, the multiplication $\star$ is given by (44).

It follows from Lemma 5.6 that $I_{1}+I_{2}-I_{3}=0$. Since the anchor $\rho$ is a Lie algebroid homomorphism, we have $I_{2}=I_{3}=0$. Thus, $I_{1}=0$, i.e.

$$
\delta_{*}\left[x_{1}, x_{2}\right]_{L_{1}}-\mathfrak{L}_{x_{1}} \delta_{*} x_{2}+\mathfrak{L}_{x_{2}} \delta_{*} x_{1}=0, \quad \forall x_{1}, x_{2} \in \Gamma\left(L_{1}\right) .
$$

Similarly, we have

$$
\delta\left[\xi_{1}, \xi_{2}\right]_{L_{2}}-\mathfrak{L}_{\xi_{1}} \delta \xi_{2}+\mathfrak{L}_{\xi_{2}} \delta \xi_{1}=0, \quad \forall \xi_{1}, \xi_{2} \in \Gamma\left(L_{2}\right) .
$$

Thus, $\left(L_{1}, L_{2}\right)$ is a left-symmetric bialgebroid.

By Theorem 5.3. we have

Corollary 5.9. Let $\left(A, A^{*}\right)$ be a left-symmetric bialgebroid. Then $\left(A^{c}, A^{* c}\right)$ is a matched pair of Lie algebroids and the bracket on $A^{c} \oplus A^{* c}$ is defined by

$$
[x+\xi, y+\eta]=[\xi, \eta]_{A^{*}}+L_{x}^{*} \xi-L_{y}^{*} \eta+L_{\xi}^{*} y-L_{\eta}^{*} x+[x, y]_{A}, \quad x, y \in \Gamma(A), \xi, \eta \in \Gamma\left(A^{*}\right) .
$$

Furthermore, $\left(A^{c} \oplus A^{* c}, \omega\right)$ is a symplectic Lie algebroid, where $\omega$ is given by (5).

By Proposition 4.13 and Proposition 5.4 , we obtain

Proposition 5.10. Let $(M, \nabla, g)$ be a pseudo-Hessian manifold. Then $\left(T M \oplus T^{*} M, \star\right.$, id + $\left.H^{\sharp},(\cdot, \cdot)_{-}\right)$is a pre-symplectic algebroid, where for all $e_{1}=x_{1}+\xi_{1}, e_{2}=x_{2}+\xi_{2} \in \Gamma\left(T M \oplus T^{*} M\right)$, the multiplication $\star$ is given by

$$
e_{1} \star e_{2}=\left(\nabla_{x_{1}} x_{2}+\mathcal{L}_{\xi_{1}} x_{2}-R_{\xi_{2}} x_{1}-\frac{1}{2} \mathrm{~d}_{*}\left(e_{1}, e_{2}\right)_{+}\right)+\left(\xi_{1} \cdot_{H} \xi_{2}+\mathcal{L}_{x_{1}} \xi_{2}-R_{x_{2}} \xi_{1}-\frac{1}{2} \mathrm{~d}\left(e_{1}, e_{2}\right)_{+}\right) .
$$

Here $\mathcal{L}$ is given by (11), $R$ is given by (23) and $(\cdot, \cdot)_{+}$is given by (14).

Assume that $\left(A, A^{*}\right)$ is a left-symmetric bialgebroid and $H \in \Gamma(A \otimes A)$. We denote by $G_{H}$ the graph of $H^{\sharp}$, i.e. $G_{H}=\left\{H^{\sharp}(\xi)+\xi \mid \forall \xi \in A^{*}\right\}$. 
Theorem 5.11. With the above notations, $G_{H}$ is a Dirac structure of the pre-symplectic algebroid $\left(A \oplus A^{*}, \star, \rho,(\cdot, \cdot)_{-}\right)$given by Proposition 5.4 if and only if $H \in \operatorname{Sym}^{2}(A)$ and the following MaurerCartan type equation is satisfied:

$$
\delta_{*} H-\llbracket H, H \rrbracket=0,
$$

where $\llbracket H, H \rrbracket$ is given by (39).

Proof. First it is easy to see that $G_{H}$ is isotropic if and only if $H \in \operatorname{Sym}^{2}(A)$. By (44), we have

$$
\begin{aligned}
& H^{\sharp}(\xi) \star \eta=\mathcal{L}_{H^{\sharp}(\xi)} \eta-\frac{1}{2} \mathrm{~d}\left\langle H^{\sharp}(\xi), \eta\right\rangle-R_{\eta} H^{\sharp}(\xi)-\frac{1}{2} \mathrm{~d}_{*}\left\langle H^{\sharp}(\xi), \eta\right\rangle ; \\
& \xi \star H^{\sharp}(\eta)=-R_{H^{\sharp}(\eta)} \xi-\frac{1}{2} \mathrm{~d}\left\langle H^{\sharp}(\xi), \eta\right\rangle+\mathcal{L}_{\xi} H^{\sharp}(\eta)-\frac{1}{2} \mathrm{~d}_{*}\left\langle H^{\sharp}(\xi), \eta\right\rangle,
\end{aligned}
$$

which implies that

$$
H^{\sharp}(\xi) \star \eta+\xi \star H^{\sharp}(\eta)=\xi \cdot H \eta+\mathcal{L}_{\xi} H^{\sharp}(\eta)-R_{\eta} H^{\sharp}(\xi)-\mathrm{d}_{*}\left\langle H^{\sharp}(\xi), \eta\right\rangle .
$$

Then by (41), we have

$$
\begin{aligned}
& \left(H^{\sharp}(\xi)+\xi\right) \star\left(H^{\sharp}(\eta)+\eta\right) \\
= & H^{\sharp}(\xi) \cdot{ }_{A} H^{\sharp}(\eta)+H^{\sharp}(\xi) \star \eta+\xi \star H^{\sharp}(\eta)+\xi \cdot A^{*} \eta \\
= & H^{\sharp}(\xi \cdot H \eta)+\mathcal{L}_{\xi} H^{\sharp}(\eta)-R_{\eta} H^{\sharp}(\xi)-\mathrm{d}_{*}\left\langle H^{\sharp}(\xi), \eta\right\rangle-\llbracket H, H \rrbracket(\xi, \cdot \eta)+\xi \cdot H \eta+\xi \cdot A^{*} \eta .
\end{aligned}
$$

Thus, $G_{H}$ is integrable if and only if for all $\xi, \eta \in \Gamma\left(A^{*}\right)$,

$$
H^{\sharp}\left(\xi \cdot A^{*} \eta\right)=\mathcal{L}_{\xi} H^{\sharp}(\eta)-R_{\eta} H^{\sharp}(\xi)-\mathrm{d}_{*}\left\langle H^{\sharp}(\xi), \eta\right\rangle-\llbracket H, H \rrbracket(\xi, \cdot, \eta) .
$$

On the other hand, we have

$$
\begin{aligned}
\delta_{*} H(\zeta, \xi, \eta)= & a_{A}(\zeta)\left\langle H^{\sharp}(\xi), \eta\right\rangle-a_{A}(\xi)\left\langle H^{\sharp}(\zeta), \eta\right\rangle \\
& -\left\langle H^{\sharp}(\xi), \zeta \cdot A^{*} \eta\right\rangle+\left\langle H^{\sharp}(\zeta), \xi \cdot A^{*} \eta\right\rangle-\left\langle[\zeta, \xi]_{A^{*}}, H^{\sharp}(\eta)\right\rangle \\
= & \left\langle H^{\sharp}\left(\xi \cdot A^{*} \eta\right)-\mathcal{L}_{\xi} H^{\sharp}(\eta)+R_{\eta} H^{\sharp}(\xi)+\mathrm{d}_{*}\left\langle H^{\sharp}(\xi), \eta\right\rangle, \zeta\right\rangle .
\end{aligned}
$$

By (48) and (49), $G_{H}$ is a Dirac structure if and only if

$$
\delta_{*} H(\zeta, \xi, \eta)-\llbracket H, H \rrbracket(\zeta, \xi, \eta)=0 .
$$

The proof is finished.

\section{References}

[1] C. Bai, Left-symmetric bialgebras and an analogue of the classical Yang-Baxter equation, Commun. Contemp. Math. 10 (2008), 221-260.

[2] D. Burde, Left-symmetric algebras, or pre-Lie algebras in geometry and physics. Cent. Eur. J. Math. 4 (2006), 323-357.

[3] B. Y. Chu, Symplectic homogeneous spaces, Trans. Amer. Math. Soc. 197 (1974) 145-159. 
[4] M. de León, J. C. Marrero and E. Martínez, Lagrangian submanifolds and dynamics on Lie algebroids, J. Phys. A 38 (2005), No. 24, R241-R308.

[5] A. Dzhumadil'daev, Cohomologies and deformations of right-symmetric algebras, J. Math. Sci. 93 (1999), 836-876.

[6] M. Gerstenhaber, The cohomology structure of an associative ring, Ann. Math. 78 (1963), $267-288$

[7] D. Iglesias, J. Marrero, D. Martin de Diego, E. Martinez and E. Padrón, Reduction of symplectic Lie algebroids by a Lie subalgebroid and a symmetry Lie group. SIGMA Symmetry Integrability Geom. Methods Appl. 3 (2007), Paper 049, 28 pp.

[8] H. Kim, Complete left-invariant affine structures on nilpotent Lie groups, J. Diff. Geom. 24 (1986), 373-394.

[9] Y. Kosmann-Schwarzbach, The Lie bialgebroid of a Poisson-Nijenhuis manifold, Lett. Math. Phys. 38 (1996), no. 4, 421-428.

[10] J. L. Koszul, Domaines bornés homogenes et orbites de groupes de transformations affines, Bull. Soc. Math. France 89 (1961), 515-533.

[11] A. Lichnerowicz and A. Medina, On Lie groups with left-invariant symplectic or Kählerian structures. Lett. Math. Phys. 16 (1988), 225-235.

[12] J. Liu, Y. Sheng, C. Bai and Z. Chen, left-symmetric algebroids. Math. Nachr. 289 (2016), No. 14-15, 1893-1908.

[13] J. Liu, Y. Sheng and C. Bai, Pre-symplectic algebroids and their applications, arXiv:1604.00146, to appear in Lett. Math. Phys.

[14] Z. Liu, A. Weinstein and P. Xu. Manin triples for Lie bialgebroids. J. Diff. Geom. 45(3) (1997), 547-574.

[15] K. C. H. Mackenzie, General theory of Lie groupoids and Lie algebroids. Lecture Note Series, 213. London Mathematical Society., Cambridge University Press, Cambridge, 2005.

[16] K. C. H. Mackenzie and P. Xu, Lie bialgebroids and Poisson groupoids. Duke Math. J. 73 (1994), 415-452.

[17] S. Majid and W. Tao, Noncommutative Differentials on Poisson-Lie groups and pre-Lie algebras, Pacific J. Math. 284 (2016), 213-256.

[18] J. Marrero, E. Padrón and M. Rodriguez-Olmos, Reduction of a symplectic-like Lie algebroid with momentum map and its application to fiberwise linear Poisson structures. J. Phys. A 45 (2012), no. 16, 165201, 34 pp.

[19] A. Medina, Flat left-invariant connections adapted to the automorphism structure of a Lie group, J. Diff. Geom. 16 (1981), 445-474.

[20] J. Milnor, Curvatures of left invariant metrics on Lie groups, Adv. Math. 21 (1976) 293-329.

[21] R. Nest and B. Tsygan, Deformations of symplectic Lie algebroids, deformations of holomorphic symplectic structures, and index theorems. Asian J. Math. 5 (2001), no. 4, 599-635. 
[22] M. Nguiffo Boyom, Cohomology of Koszul-Vinberg algebroids and Poisson manifolds. I. Banach Center Publ. 54 (2001), 99-110.

[23] M. Nguiffo Boyom, KV-cohomology of Koszul-Vinberg algebroids and Poisson manifolds. Internat. J. Math. 16 (2005), no. 9, 1033-1061.

[24] X. Ni and C. Bai, Pseudo-Hessian Lie algebras and L-dendriform bialgebras, J. Algebra. 400 (2014) 273-289.

[25] H. Shima, Homogeneous Hessian manifolds, Ann. Inst. Fourier(Grenoble) 30 (1980), 90-128.

[26] H. Shima, The geometry of Hessian structures. World Scientific Publishing Co. Pte. Ltd., Hackensack, NJ, 2007. xiv+246 pp.

[27] E. B. Vinberg, Convex homogeneous cones, Transl. Moscou Math. Soc. 12 (1963), 340-403. 